\title{
Visible and NIR image fusion using weight-map-guided Laplacian-Gaussian pyramid for improving scene visibility
}

\author{
ASHISH V VANMALI* and VIKRAM M GADRE \\ Department of Electrical Engineering, Indian Institute of Technology, Bombay, Mumbai 400076, India \\ e-mail: ashishvanmali@iitb.ac.in; vanmaliashish@gmail.com; vmgadre@ee.iitb.ac.in
}

MS received 20 November 2015; revised 19 September 2016; accepted 2 December 2016

\begin{abstract}
Image visibility is affected by the presence of haze, fog, smoke, aerosol, etc. Image dehazing using either single visible image or visible and near-infrared (NIR) image pair is often considered as a solution to improve the visual quality of such scenes. In this paper, we address this problem from a visible-NIR image fusion perspective, instead of the conventional haze imaging model. The proposed algorithm uses a LaplacianGaussian pyramid based multi-resolution fusion process, guided by weight maps generated using local entropy, local contrast and visibility as metrics that control the fusion result. The proposed algorithm is free from any human intervention, and produces results that outperform the existing image-dehazing algorithms both visually as well as quantitatively. The algorithm proves to be efficient not only for the outdoor scenes with or without haze, but also for the indoor scenes in improving scene visibility.
\end{abstract}

Keywords. Image dehazing; Laplacian-Gaussian pyramid; multi-resolution fusion; visible-NIR image fusion; weight map.

\section{Introduction}

Visibility and colour of an image are greatly affected by bad weather such as haze, fog, smoke, aerosol, etc. The attenuation and the smoothing effect as a result of scattering due to such conditions result in loss of contrast and details, and at the same time changes the colour perception of the camera while capturing image. Recovering the actual scene information is a challenge and is often termed as dehazing.

The traditional methods like gamma correction and histogram equalization provide a limited enhancement of such images and at some points their results can look more artificial. Some researchers have proposed algorithms that make use of multiple images of the same scene under different weather conditions to enhance visibility [1]. Some dehazing algorithms make use of multiple images captured at different degrees of polarization [2,3]. In both these cases, the algorithms are not suitable to handle dynamic scenes and are highly sensitive to the way in which these multiple images are captured.

In the last decade, many algorithms based on singleimage dehazing have been proposed by different researchers [5-10]. These algorithms estimate airlight and transmission based on certain assumptions or inputs from the user. The main limitation of these single-dehazing algorithms is that, the details that are not registered in the

*For correspondence image cannot be restored and are lost completely, no matter how good the performance of the algorithm is. Also, most single-image dehazing algorithms proposed in the literature require human intervention and are computationally complex.

To tackle this issue, dehazing based on the combination of visible and near-infrared (NIR) image has become popular in recent times. Digital camera sensors can capture visible spectrum $(400-700 \mathrm{~nm})$ as well as NIR spectrum (700-1100 nm). To capture a visible image, an infrared filter popularly called hot mirror is placed in front of camera sensors to prevent NIR light falling on the sensor. Removing the hot mirror will capture visible and NIR spectrum at the same time. To capture NIR image, it is necessary to put a visible light blocking filter on the top. For complete details of NIR image acquisition, readers are referred to Fredembach and Süsstrunk [11], Zhang et al [12], Zhuo et al [13] and Sadeghipoor et al [14].

The NIR images so captured exhibit high contrast. Also, NIR can see through haze and it gives bright response for vegetation and clouds. These features of NIR images become handy to cater to dehazing problem. The pair of visible-NIR images is used by some researchers [15] to estimate airlight and transmission. Algorithms without estimation of haze or airlight, with a pair of visible-NIR images, have also been proposed in literature $[12,13,16]$.

In this paper, we propose a mechanism to fuse visible and NIR images. The multi-resolution fusion process is 
guided by weight maps generated using the characteristics of visible and NIR images, so that the final fused result has enhanced details, contrast and is vibrant in colour. An example is demonstrated in figure 1 . The algorithm is fully automatic and is less computationally demanding. It is capable of handling both outdoor and indoor scenes. The results produced by this algorithm are also superior in quantitative analysis.

\section{Related work}

The optical model used for representing image degradation due to hazy conditions consists of direct attenuation and airlight as follows $[5,7,8]$ :

$$
\mathbf{I}(x, y)=\mathbf{J}(x, y) t(x, y)+\mathbf{A}(1-t(x, y))
$$

where, $\mathbf{I}(x, y)$ is the observed image, $\mathbf{J}(x, y)$ is the haze-free image, $\mathbf{A}$ is the global atmospheric light (also called as
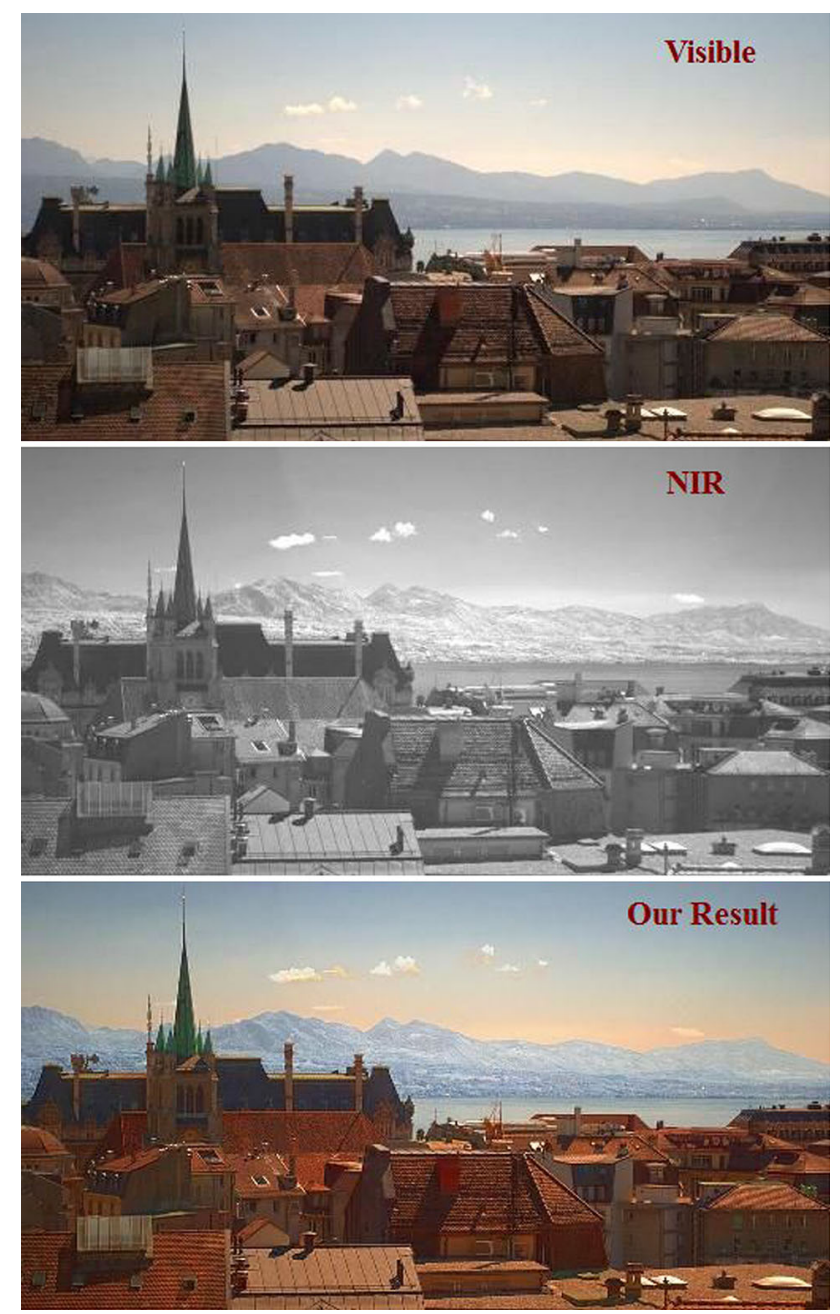

Figure 1. Demonstration of visible-NIR image fusion: note the improvement in the details and contrast of the visible image with vibrant colour perception for our result. (Images courtesy: EPFL database EPFL [4].) airlight colour) and $t(x, y)$ is the medium transmission describing the portion of the light that is not scattered and reaches the camera for the image pixel $(x, y)$. The term $\mathbf{J}(x, y) t(x, y)$ is called as direct attenuation and the term $\mathbf{A}(1-t(x, y))$ is called as airlight. The transmission $t(x, y)$ is defined as

$$
t(x, y)=e^{-\beta d(x, y)}
$$

where, $\beta$ is the scattering coefficient of the atmosphere and $d(x, y)$ is the scene depth. Recovering a haze-free image $\mathbf{J}$ from $\mathbf{I}$ needs estimation of $\mathbf{A}$ and $t$, which is an ill-posed problem and correct assumptions play an important role in getting good results. Most popular dehazing algorithms are based on this haze imaging model.

One of the popular algorithms in single-image dehazing was proposed by Fattal [5]. Fattal's approach is based on the assumption that the shading and transmission functions are locally statistically uncorrelated. His model accounts for surface shading along with the scene transmission. It finds constant-albedo regions and resolves the airlightalbedo ambiguity using the constraint of surface shading. The airlight colour is also estimated using the uncorrelation principle. It recovers haze-free scene contrast, but its performance greatly depends on the statistics of the input data. The assumption fails in cases of very dense haze.

Tan's [8] work is based on two assumptions: first, images with enhanced visibility have more contrast than hazy images; second, airlight has a smooth variation depending on the distance of objects to the viewer. The image is dehazed by maximizing the local contrast of the restored image in the framework of Markov random fields (MRFs). The results tend to have over-saturated colours. Also, it has halo artifact at depth discontinuities.

He et al [7] proposed a dark channel prior to remove haze from a single-input image. The dark channel prior is based on the statistics of outdoor haze-free images. According to this prior, most local patches in outdoor hazefree images contain some pixels whose intensity is very low in at least one colour channel. Based on this prior and the haze imaging model as described in Eq. (1), they directly estimate haze and depth map, which is then used to recover a dehazed image. The method depends on the haze imaging model, and may fail when this model is physically invalid. Also, the method may fail to recover the true scene radiance of the distant objects as it assumes common transmission for all colour channels. A few researchers [17, 18] have extended the use of the dark channel prior for dehazing remotely sensed images.

Zhang et al [10] reconstructed a haze-free image through haze layer estimation based on the image filtering approach using both low-rank technique and the overlap averaging scheme. Guo et al [6] considered the haze as the veil layer instead of transmission. They employed the retinex algorithm and the depth information of the original image to remove the veil layer. Lan et al [19] proposed a three-stage 
algorithm for haze removal, considering sensor blur and noise. It involved elimination of blur/noise interference and estimation of transmission and atmospheric light by the dark channel prior, followed by non-local regularization method to dehaze the image.

Fattal [20] proposed a new method based on colour-lines. According to colour-lines, there is a generic regularity in natural images in which pixels of small image patches typically exhibit one-dimensional distributions in RGB colour space. The scene transmission for the hazy images was derived from the lines' offset from the origin. A MRF model was used to resolve ambiguities due to noisy and scattered estimates for producing complete and regularized transmission map.

Most of these previous works and works by other researchers on single image dehazing are computationally expensive. Tarel and Hautière [9] proposed a fast visibility restoration mechanism based on a filtering approach. More recently, another fast mechanism based on colour attenuation prior based on the difference between the brightness and the saturation of the pixels within the hazy image was proposed by Zhu et al [21]. They modelled the scene depth with the brightness and the saturation of the hazy image using a supervised learning method. The depth estimation was used for transmission recovery followed by restoring scene radiance.

Accurate estimation of the atmospheric light is another challenging issue in dehazing, which directly affects the visual appearance of the dehazed image. Sulami et al [22] proposed an automatic method to recover atmospheric light from hazy images. The magnitude of the atmospheric light was estimated using maximum-brightness transmission invariance prior. The orientation of the atmospheric light was recovered using a simplified model that describes simple geometric configurations of pixels inside small mono-albedo patches.

Ancuti and Ancuti [23] proposed a fusion-based strategy that used two hazy image inputs obtained by applying a white balance and a contrast enhancement. They used luminance, chromaticity and saliency features for weight maps. In another work, Ancuti and Ancuti [24] used a lightness predictor for contrast enhancement for the regions with less chromatic contrast, while preserving original colour spatial distribution and the local contrast. Also, they performed an image matching evaluation for hazy images.

The key limitation of any single image dehazing algorithm is the details that are not captured in RGB spectrum because of scattering, which cannot be recovered back. To reduce the computational complexity and also to improve the overall results of the single-image dehazing algorithms, algorithms based on the combination of visible and NIR image have become popular in recent times. The NIR images retain more details on distant objects than the corresponding colour image, since NIR can penetrate through hazy conditions as opposed to visible band $[11,15,16]$. This simplifies the problem of dehazing as more details are available due to NIR image.
Feng et al [15] proposed a two-stage dehazing method, exploiting the dissimilarity between visible and NIR images for airlight colour estimation, followed by a dehazing procedure through an optimization framework. Using the haze model in Eq. (1), they enhanced the details based on the transmission map and also recovered the colour.

The enhancement process proposed by Zhang et al [12] uses the Haar wavelet decomposition, and comprises three major steps: computing the weighted region mask, transferring contrast and transferring texture. The visible and the NIR image pair is first decomposed into average and detail wavelet subbands. The contrast in the average subband and the texture in the detail subbands are transferred using weighted region mask from NIR image to the visible image.

An edge-preserving multi-resolution decomposition based on the Weighted Least Squares (WLS) optimization framework is presented by Schaul et al [16]. A pixel level fusion criterion that maximizes contrast was employed to obtain haze-free image using visible and NIR images.

We propose a three-stage dehazing scheme: first, calculating weight maps using measures of local entropy, local contrast and visibility. Second, fusing visible and NIR images guided by weight maps. Third, a post-processing step for colour and sharpness correction(CSC). This algorithm does not depend on the haze imaging model, thus eliminating the need for estimation of haze or airlight. Also, the algorithm will be applicable in case of images without sky or indoor images, where the haze imaging model is invalid.

\section{Proposed algorithm}

Mertens et al [25] has demonstrated the effectiveness of a weight-map-guided multi-resolution fusion in the context of multi-exposure image fusion. In the line of their work, we propose the algorithm for the fusion of visible and NIR images. The visible image has three colour channels, viz., $\mathrm{R}, \mathrm{G}$ and $\mathrm{B}$, whereas NIR image is a single-channel image. Therefore, we cannot combine visible and NIR image directly. Also, replacing either R, G or B colour channel with NIR is not a suitable choice [11]. Instead, combining NIR with luminance component gives more pleasing results. Luminance-chrominance colour encodings, such as HSV, YCbCr, YUV or L*a*b*, are the potential candidates to incorporate NIR information. We choose HSV over other colour spaces, since HSV has luminance decoupled from colour information and preserves colour saturation better than others [11].

To fuse visible and NIR images, we first convert the visible image from RGB to HSV colour space. The value of hue and saturation is kept intact and we fuse the luminance component of the visible image with that of NIR image to form the fused luminance component. The fused luminance component along with hue and saturation is then converted back from HSV colour space to RGB colour space. A post- 
processing step of CSC is employed to form the final result. Figure 2 demonstrates the work flow of our algorithm.

\subsection{Weight map generation}

The weight maps play a critical role in the outcome of the final fused result. The weight maps generated should have a non-negative value and should lie in the range of $[0,1]$. The weight should sum up to 1 at each pixel. One needs to keep in mind the characteristics of the visible and NIR images while selecting measures to generate the weight maps. We use the following measures to generate the weight maps.

3.1a Normalized local entropy: The aim of fusion algorithm is to transfer maximum information from visible and NIR image to that of fused image. To ensure this, we introduce a weight measure of normalized local entropy $H$ calculated over a local neighbourhood $\eta$ around a pixel $(x, y)$ as

$$
H(x, y)=-\frac{1}{8} \sum_{(i, j) \in \eta} p(f,(i, j)) \log p(f,(i, j))
$$

where, $p(f)$ is the probability of occurrence of $f^{\text {th }}$ grey level. Since both visible and NIR images have 8 bits per pixel per

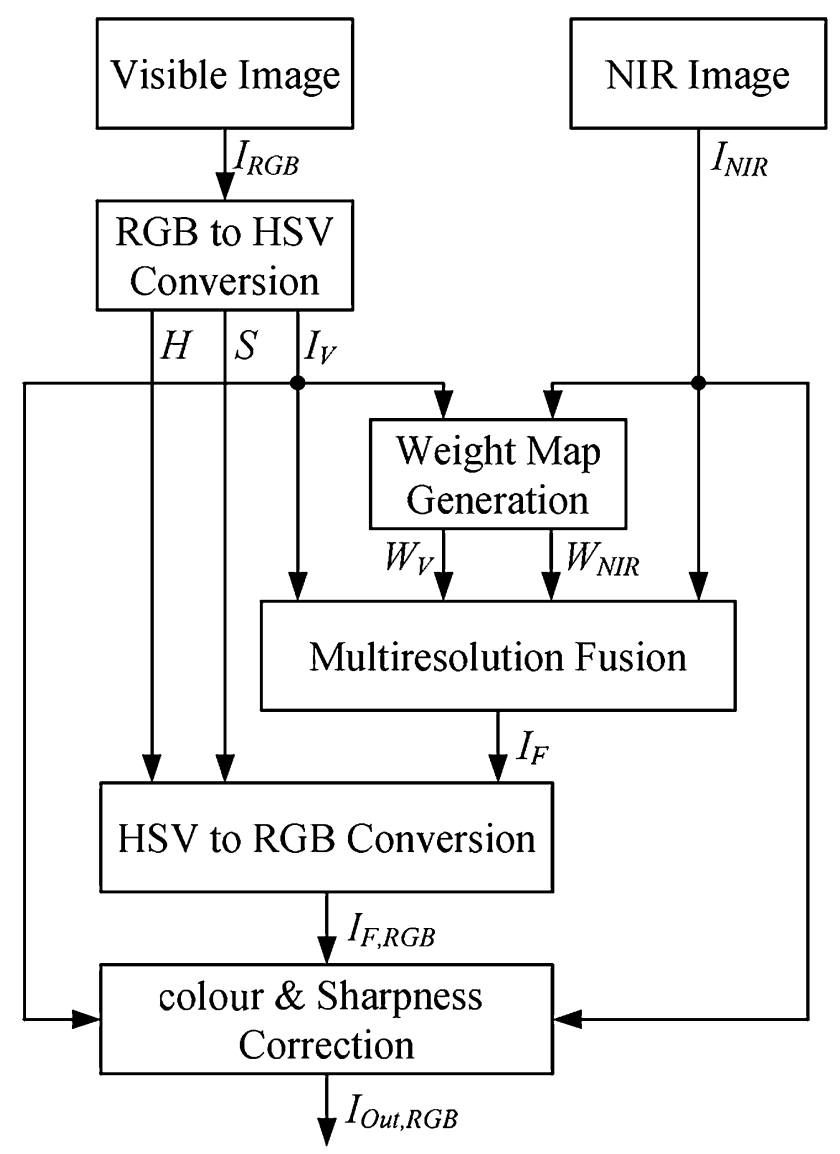

Figure 2. Work flow of our algorithm. channel, a normalization factor of $\frac{1}{8}$ is used to ensure that the value of $H$ lies in the range $[0,1]$. In our implementation, we use a fixed neighbourhood of size $5 \times 5$.

3.1b Local contrast: NIR images exhibit overall higher contrast than that of visible image. The response of NIR is saturated (bright) in case of vegetation, clouds, etc. and is desaturated (dark) for clear sky, water, etc., which makes the overall contrast of the NIR image high. Also, NIR has different responses to different colours. This indicates that just the value of the NIR channel cannot be used as a measure for the fusion. If used directly, it will produce the results that are either washed out or too dark instead of producing the details in such regions. To avoid this scenario, one has to also take into account the local activity around the pixel. We use a measure of local contrast that weights the pixels based on the local activity around it.

The local contrast $C$ over a local neighbourhood $\eta$ around a pixel $(x, y)$ is calculated as

$$
C(x, y)=\sqrt{\frac{1}{N} \sum_{(i, j) \epsilon \eta}(I(i, j)-\mu(x, y))^{2}}
$$

where, $\mu(x, y)$ is the local mean given by

$$
\mu(x, y)=\frac{1}{N} \sum_{(i, j) \in \eta} I(i, j)
$$

and $N$ is the number of pixels in the local neighbourhood $\eta$. If pixels in the neighbourhood exhibit more deviation from the local mean, it indicates the presence of activity in that region and is eventually given a higher weight and viceversa. In our implementation, we use a fixed neighbourhood of size $5 \times 5$.

3.1c Visibility: NIR can be transparent or nontransparent based on the medium. It can see through haze and fog where visible spectrum cannot. At the same time, it is opaque through water where visible spectrum can see through up to a certain extent. Hence, it is quite obvious that one should assign weights to visible and NIR images based on their capacity in capturing details underneath a particular medium. We define a visibility metric using a local blur estimation method. First, we blur the image using a Gaussian function with known standard deviation. Then root square blurred difference between image and its blurred version is used as a visibility measure $V$ as follows:

$$
I_{b l u r}(x, y)=I(x, y) \otimes g_{M}\left(x, y, \sigma_{1}\right)
$$

and

$$
V(x, y)=\sqrt{\left(I(x, y)-I_{b l u r}(x, y)\right)^{2} \otimes g_{M}\left(x, y, \sigma_{2}\right)} .
$$

Here, $g_{M}(x, y, \sigma)$ is a $2 \mathrm{D}$ circularly symmetric Gaussian weighting function with a $M \times M$ window and standard deviation $\sigma$. The operator $\otimes$ indicates 2D convolution 
operation. In our implementation, we use $M=5$ and $\sigma_{1}=\sigma_{2}=2$.

For each pixel, we combine these three weights to form a single weight as

$$
W_{i}(x, y)=H_{i}(x, y)^{\alpha_{1}} \times C_{i}(x, y)^{\alpha_{2}} \times V_{i}(x, y)^{\alpha_{3}}
$$

The subscript $i$ indicates channel, either visible or NIR. The product is used to combine these measures over a linear combination. The product acts as an AND operation instead of OR operation as in the case of linear combination. The weighting exponents $\alpha_{1}, \alpha_{2}$ and $\alpha_{3}$ take values in $[0,1]$ and control the contribution of each weight measure in the final fused result. For more details on weight map generation, readers can refer to the work of Mertens et al [25]. We set $\alpha_{1}=\alpha_{2}=\alpha_{3}=1$, so that each measure contributes equally to the final fused result. The weights are then normalized so that they sum to 1 at each pixel as

$$
\begin{gathered}
W_{V_{N}}(x, y)=\frac{W_{V}(x, y)}{W_{V}(x, y)+W_{N I R}(x, y)} \\
W_{N I R_{N}}(x, y)=\frac{W_{N I R}(x, y)}{W_{V}(x, y)+W_{N I R}(x, y)}
\end{gathered}
$$

Figure 3 demonstrates an example of weight maps generation and their effect on the final fused result. For the visible image, the details underneath water are visible; at the same time the top background is desaturated in colour. For NIR image, the details underneath water are not visible, but the waves on the water surface are seen. The vegetation in the top background is bright in the NIR image. When we employ only normalized local entropy as a measure, it will try to put all the information from both the images to the final fused result. This increases saturation of the background, but also gives a wash-out effect to water details. Local contrast shows the improvement in texture for the background with a slight improvement in the water details. Visibility shows the details of the water best preserved compared with local entropy and local contrast, but suffers a degradation in detail transfer for vegetation compared with local contrast. The combination of three weights together gives advantages of all three combined together, thus exhibiting details of both water and background well preserved with increase in saturation.

\subsection{Multi-resolution fusion}

The weight maps generated for visible and NIR images are not smooth. Hence, one cannot fuse visible and NIR images simply by taking a weighted sum using these weight maps. We use the combination of Laplacian and Gaussian pyramids as used by Mertens et al [25] to accomplish the fusion.
The visible and NIR images are decomposed using a Laplacian pyramid, and the normalized weight maps are decomposed using a Gaussian pyramid.

Mathematically the $l^{\text {th }}$ level of Gaussian pyramid is represented as

$$
G\{I(x, y)\}_{l}=\left[g(x, y) \otimes G\{I(x, y)\}_{l-1}\right]_{\downarrow 2}
$$

where, $g(x, y)$ is the Gaussian kernel, $G\{I(x, y)\}_{l-1}$ is the approximation at the previous level and $\downarrow 2$ is down-sampling operation by 2 . Similarly, the Laplacian pyramid decomposition can be represented as

$$
L\{I(x, y)\}_{l}=G\{I(x, y)\}_{l-1}-R(x, y) \otimes\left[G\{I(x, y)\}_{l}\right]_{\uparrow 2}
$$

where, $L\{I(x, y)\}_{l}$ is the Laplacian decomposition at level $l$, $R(x, y)$ is the interpolation filter and $\uparrow 2$ is upsampling operation by 2 .

The fused Laplacian pyramid is then obtained by combining the Laplacian pyramids at each level of decomposition weighted by corresponding Gaussian pyramids as

$$
\begin{aligned}
L\left\{I_{F}(x, y)\right\}_{l} & =G\left\{W_{V_{N}}(x, y)\right\}_{l} L\left\{I_{V}(x, y)\right\}_{l} \\
& +G\left\{W_{N I R_{N}}(x, y)\right\}_{l} L\left\{I_{N I R}(x, y)\right\}_{l} .
\end{aligned}
$$

The fused pyramid $L\left\{I_{F}(x, y)\right\}_{l}$ is then collapsed to obtain $I_{F}(x, y)$, which is the fused image of the luminance component of the visible image with that of NIR image. For smooth blending, the pyramidal decomposition is carried out up to the level of 1 pixel.

\section{$3.3 C S C$}

The fused luminance component along with hue and saturation are then converted back from HSV colour space to RGB colour space to form the fused colour image $I_{F, R G B}(x, y)$. Since, the original luminance component $I_{V}(x, y)$ is replaced by fused luminance component $I_{F}(x, y)$, it also alters the overall colour composition of the image when converted back to RGB colour space from HSV colour space. Also, the images with saturated colour are more vivid. Hence, we introduce a colour correction mechanism that operates like a tone mapping operator for the fused colour image. The result is a colour image that has increased saturation and looks vibrant in colour. The colour correction is described as

$$
I_{T, R G B}^{c}(x, y)=\left(\frac{I_{F, R G B}^{c}(x, y)}{I_{F}(x, y)}\right)^{\beta}\left(\frac{I_{V}(x, y)+I_{N I R}(x, y)}{2}\right)
$$

for $c=R, G$ and $B$ colour components. The exponent $\beta$ is the tone control parameter and is greater than or equal to one. It controls the colour saturation of the resultant image. Larger the value of $\beta$, larger the saturation. In our 

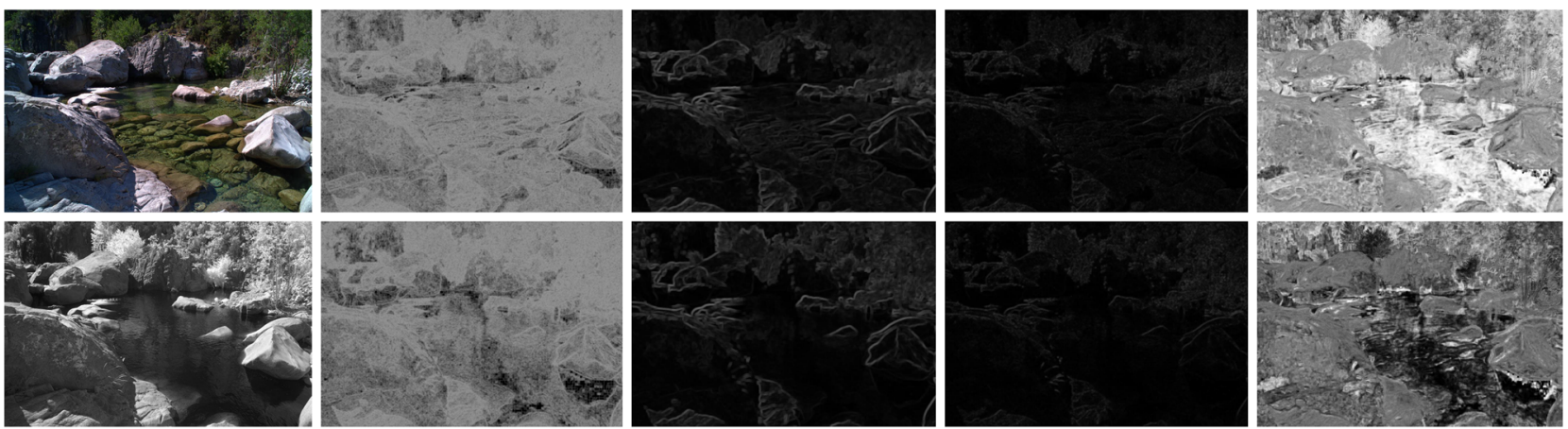

(a)
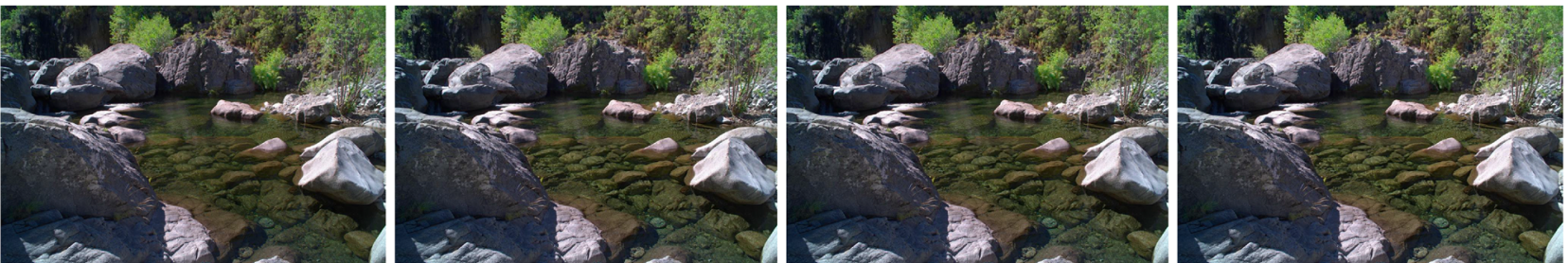

(b)
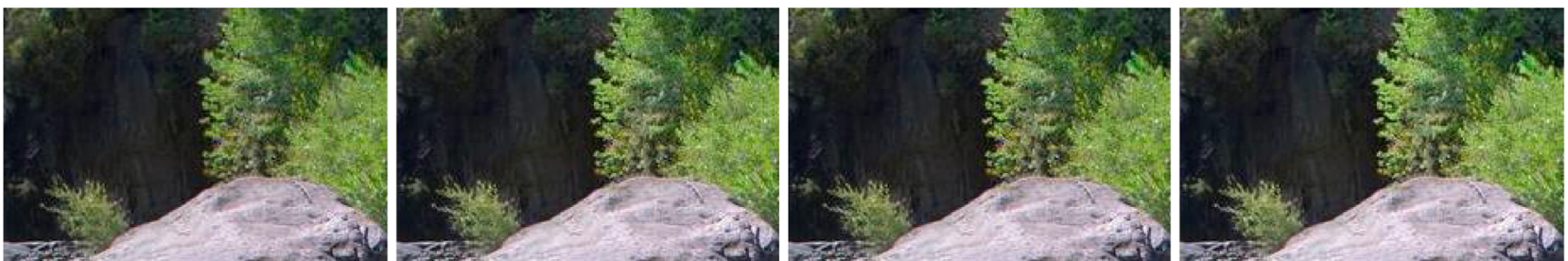

(c)
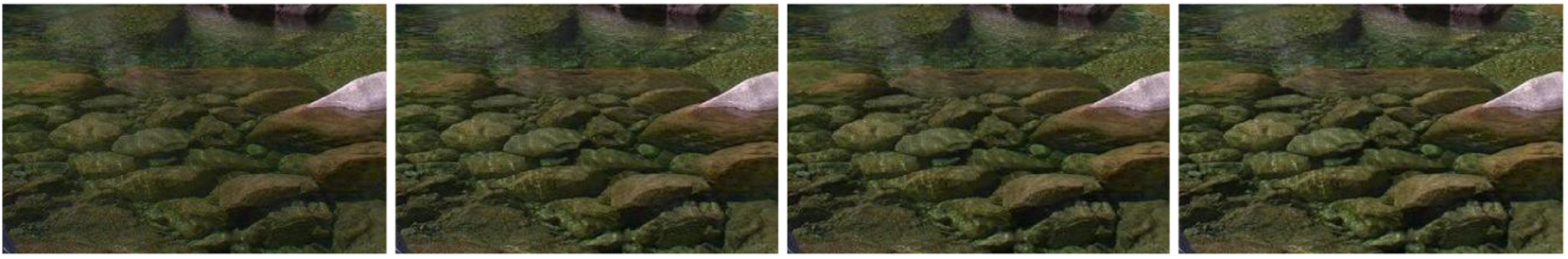

(d)

Figure 3. Demonstration of weight maps and their effect on the final fused result. (Images courtesy: EPFL database EPFL [4].) (a) Columns-wise from left to right: input visible-NIR image pair, normalized local entropy, local contrast, visibility and final weight maps. (b) Fusion results. From left to right: using only normalized local entropy, using only local contrast, using only visibility and using final weight maps. (c) Cropped portions showing vegetation part of fusion results. From left to right: using only normalized local entropy, using only local contrast, using only visibility and using final weight maps. (d) Cropped portions showing water part of fusion results. From left to right: using only normalized local entropy, using only local contrast, using only visibility and using final weight maps.

implementation, we use a fixed $\beta=1.5$. User can tune this parameter to obtain the desired level of colour saturation.

The multi-resolution fusion, as described earlier, results in smoothing of the fused image. Hence, we employ a sharpening mechanism [26] to improve the visual quality of the fused image. The colour-corrected image $I_{T, R G B}(x, y)$ is again transformed to HSV colour space. The luminance component $I_{T}(x, y)$ is first filtered by a high-pass filter, which extracts the high-frequency components, and then a scaled version of this high-pass filter output is added back to $I_{T}(x, y)$ : 


$$
I_{\text {Out }}(x, y)=I_{T}(x, y)+\lambda\left[I_{T}(x, y) \otimes F(x, y)\right]
$$

The image is again converted back to RGB colour space to form the final result $I_{O u t, R G B}(x, y)$. Here, $F(x, y)$ indicates a high-pass filter, and $\lambda$ is a sharpening control parameter greater than or equal to zero. We set $\lambda=0.4$ in our work. Linear high-pass filters can lead to unacceptable results if the original image is noisy. Instead, weighted median (WM) filters produce sharpening with better noise immunity. We use the following WM filter mask for high-pass filter commonly found in the literature [26]:

$$
W M=\frac{1}{3}\left[\begin{array}{rrr}
-1 & -1 & -1 \\
-1 & 8 & -1 \\
-1 & -1 & -1
\end{array}\right]
$$

The CSC does not improve the visibility of the output image, but enhances the visual quality. The output with CSC is more vivid as it has less blur and the colours are more saturated. If required, the user can treat this step of CSC as optional. We demonstrate our results without CSC as well as with CSC.

\section{Results and discussion}

To demonstrate the effectiveness of the proposed method, we used EPFL database EPFL [4], which consists of more than 450 images with nine different scene categories. All visible and NIR images used are of $1024 \times 680$ resolution and are spatially registered. In the experiments, we performed algorithms by executing the MATLAB code on a laptop with $2.4 \mathrm{GHz}$ Intel i5 Processor and $4 \mathrm{~GB}$ RAM. The experiments are performed with and without CSC. The average execution time for the proposed algorithm is approximately $3.19 \mathrm{~s}$ without CSC and it takes approximately $3.77 \mathrm{~s}$ with CSC when tested for 360 runs (72 datasets, each executed five times).

The results of the proposed algorithm are compared with the state-of-the-art single/multiple image-dehazing algorithms. For single-image dehazing, results of He et al [7], Tarel and Hautiére [9], Ancuti and Ancuti [23] and Zhu et al [21] are used for comparison. For visible-NIR-based dehazing, results of Zhang et al [12] and Schaul et al [16] are used for comparison. The results are demonstrated in figures 4, 5, 6, 7, 8 and 9. We have used the MATLAB codes of these algorithms available online with their predefined settings. The average execution times for these algorithms were also recorded for 360 runs (72 datasets, each executed 5 times). Comparisons of these execution times with our algorithm are presented in table 1. It indicates that our algorithm with or without CSC is faster compared with all the algorithms used in the comparison.

For the brevity of space, we demonstrate the results for 12 datasets with different conditions and incorporating all nine categories of the database. The first 4 datasets represent images with hazy condition. Datasets 1 and 2 are of category 'country', first indicating white clouds and second with dark clouds with overcast conditions. Dataset 3 is of the category 'mountain' representing a natural scene. Dataset 4 is of 'urban' category. Datasets 5-8 represent haze-free images with clear sky. Dataset 5 is of 'field' category whereas dataset 6 is from the 'old building' category. Dataset 7 is of form 'street' category and dataset 8 is from the 'water' category. Datasets 9 and 10 are haze-free images without sky. Here, dataset 9 is again from the 'old building' category and dataset 10 is from the 'forest' category. Dataset 11 exhibits transparent and non-transparent behaviour of visible and NIR through water and is from the category 'water'. Dataset 12 exhibits an indoor image from category 'indoor'. For datasets $1-8$, the haze imaging model holds true; however, it is invalid for datasets 9-12.

The results of He et al [7] and Tarel and Hautière [9] increase the contrast in the hazy images. In case of hazefree images, He et al do not add any significant improvement, whereas in images where the sky is not visible or in indoor image, it gives a dark appearance to the results. In many cases, He et al produce over-saturated colour, making the results look artificial. Tarel and Hautière, on the other hand, shows significant improvement in the appearance with well-saturated colours. Tarel and Hautière [9] yield more details than those of $\mathrm{He}$ et al [7]. One needs to tune the processing parameters for these algorithms based on the images to obtain a good appearance.

The results of Ancuti and Ancuti [23] show similar performance to that of Tarel and Hautière [9] for hazy images, but lack in colour reproduction. In other cases, their results have bright appearance, which leads to loss of details in certain areas when compared with other results. For indoor images, Ancuti and Ancuti have better contrast and good colour reproduction compared with other algorithms. Zhu et al [21] give consistent performance similar to that of Tarel and Hautière [9] for hazy as well as haze-free images with good amount of details and colour output. For distant objects, Zhu et al [21] have more details compared with $\mathrm{He}$ et al [7], Tarel and Hautière [9] and Ancuti and Ancuti [23]. For the images without sky and indoor images, Zhu et al [21] show a dark appearance to the output.

Zhang et al [12] and Schaul et al [16] show an increment in detail transfer compared with those with $\mathrm{He}$ et al [7], Tarel and Hautière [9], Ancuti and Ancuti [23] and Zhu et al [21], which are mainly contributed by NIR images. The results of Schaul et al [16] exhibit better detail transfer in hazy conditions as opposed to haze-free conditions, while exactly opposite behaviour is seen in case of results of Zhang et al [12], when compared with each other. The performance in Schaul et al [16] is inferior in case of indoor image and that without sky. Zhang et al [12] show better colour saturation as compared with that of Schaul et al [16], but it is less compared with that of He et al [7], Tarel and Hautière [9] and Zhu et al [21]. 

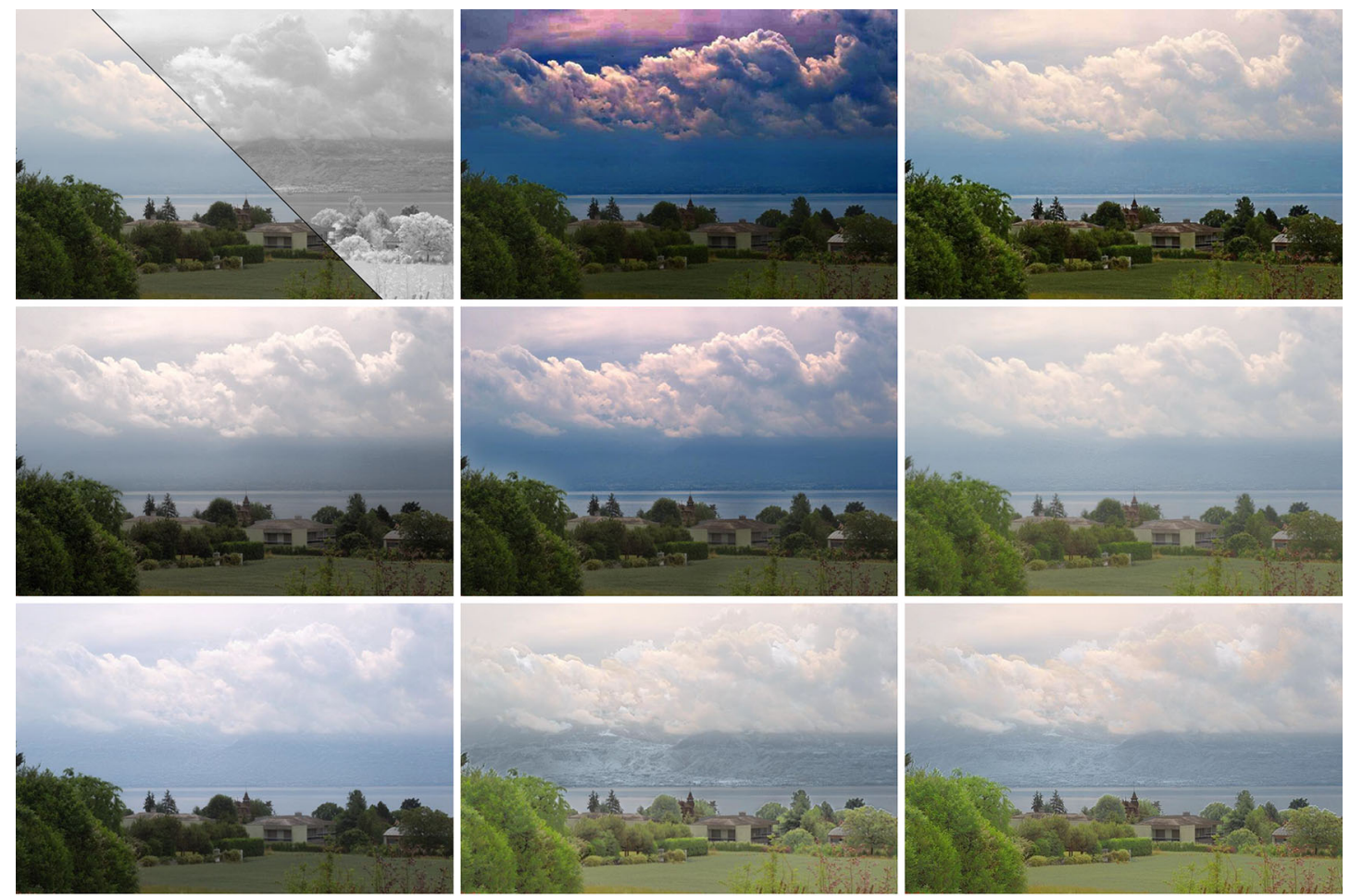

(a)
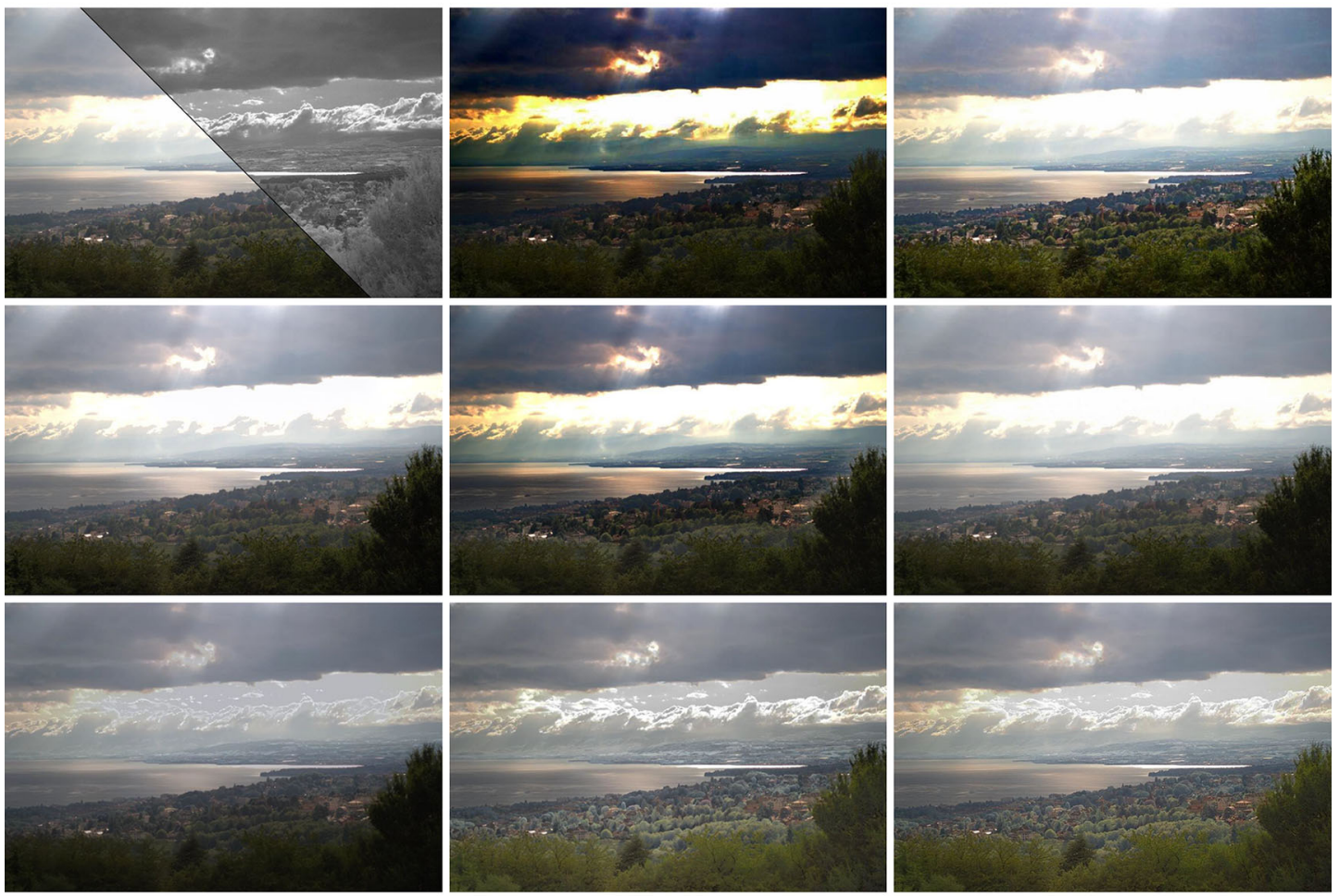

(b)

Figure 4. Demonstration of results for hazy conditions. For each dataset, 1st row left to right: visible-NIR image pair, results of He et al [7] and results of Tarel and Hautière [9]; 2nd row left to right: results of Ancuti and Ancuti [23], results of Zhu et al [21] and results of Zhang et al [12]; 3rd row left to right: results of Schaul et al [16], our result without CSC and our results with CSC. (Images courtesy: EPFL database EPFL [4].) (a) Dataset 1, (b) Dataset 2. 

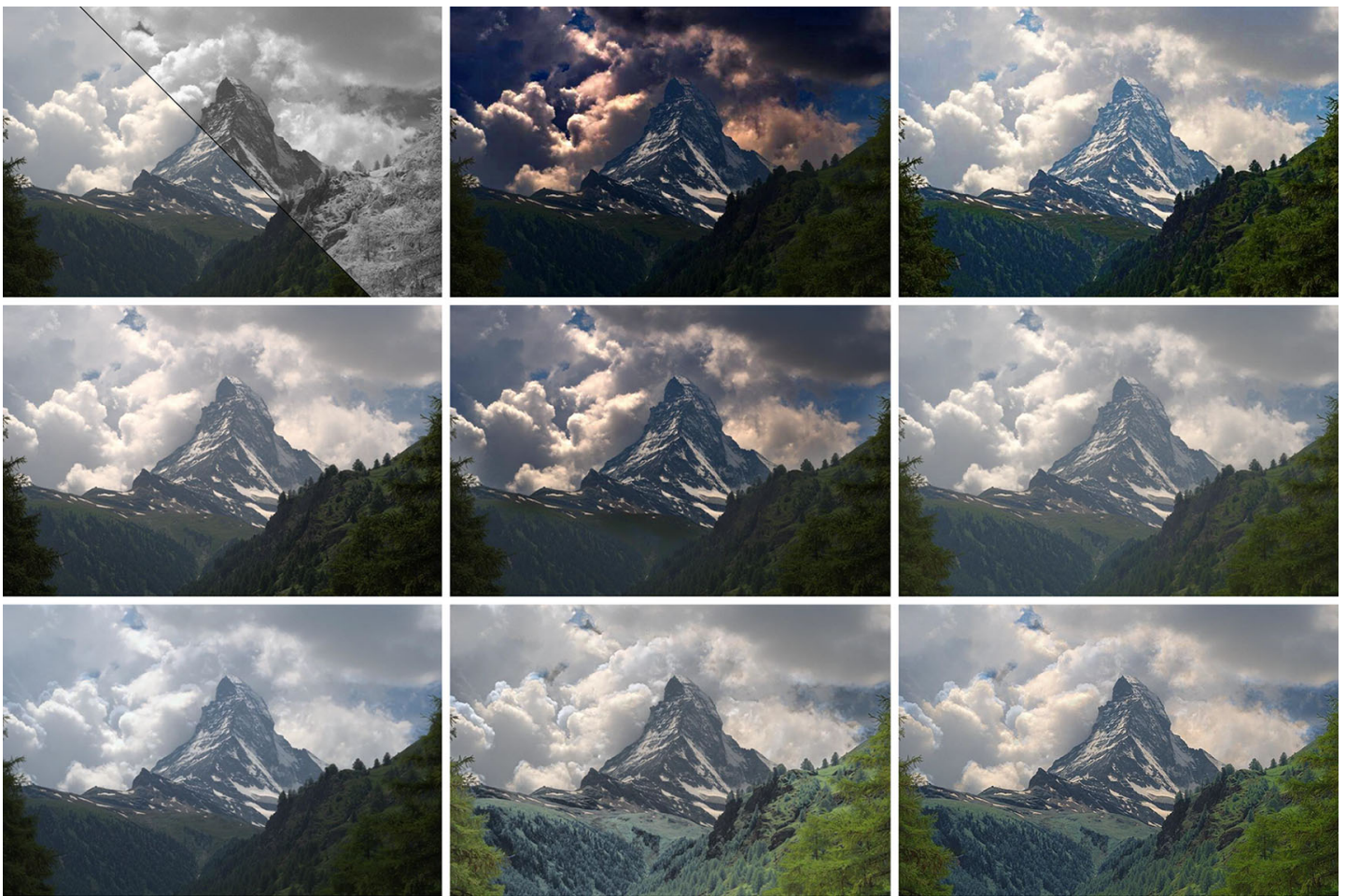

(a)
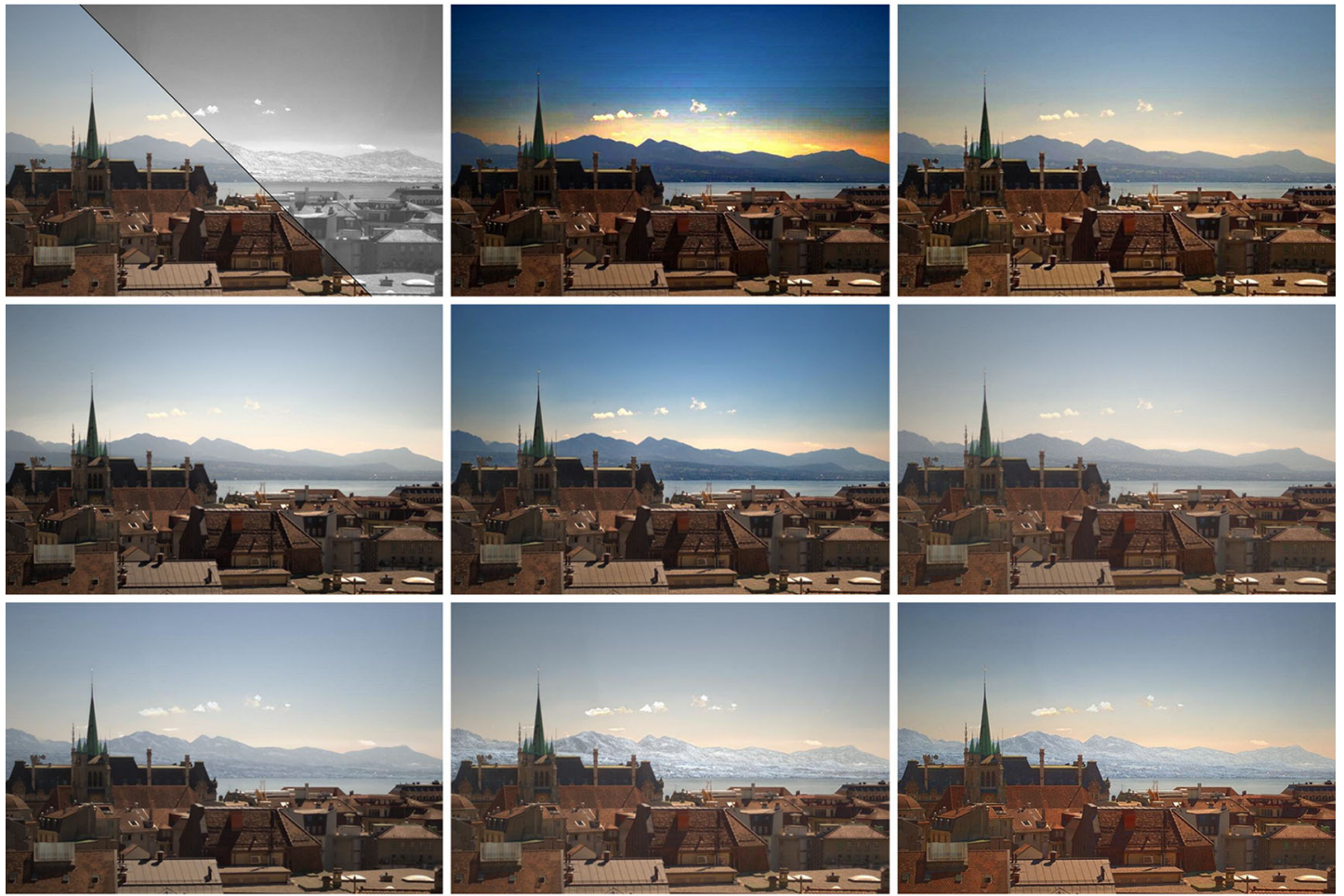

(b)

Figure 5. Demonstration of results for hazy conditions. For each dataset, 1st row left to right: visible-NIR image pair, results of He et al [7] and results of Tarel and Hautière [9]; 2nd row left to right: results of Ancuti and Ancuti [23], results of Zhu et al [21] and results of Zhang et al [12]; 3rd row left to right: results of Schaul et al [16], our result without CSC and our results with CSC. (Images courtesy: EPFL database EPFL [4].) (a) Dataset 3, (b) Dataset 4. 

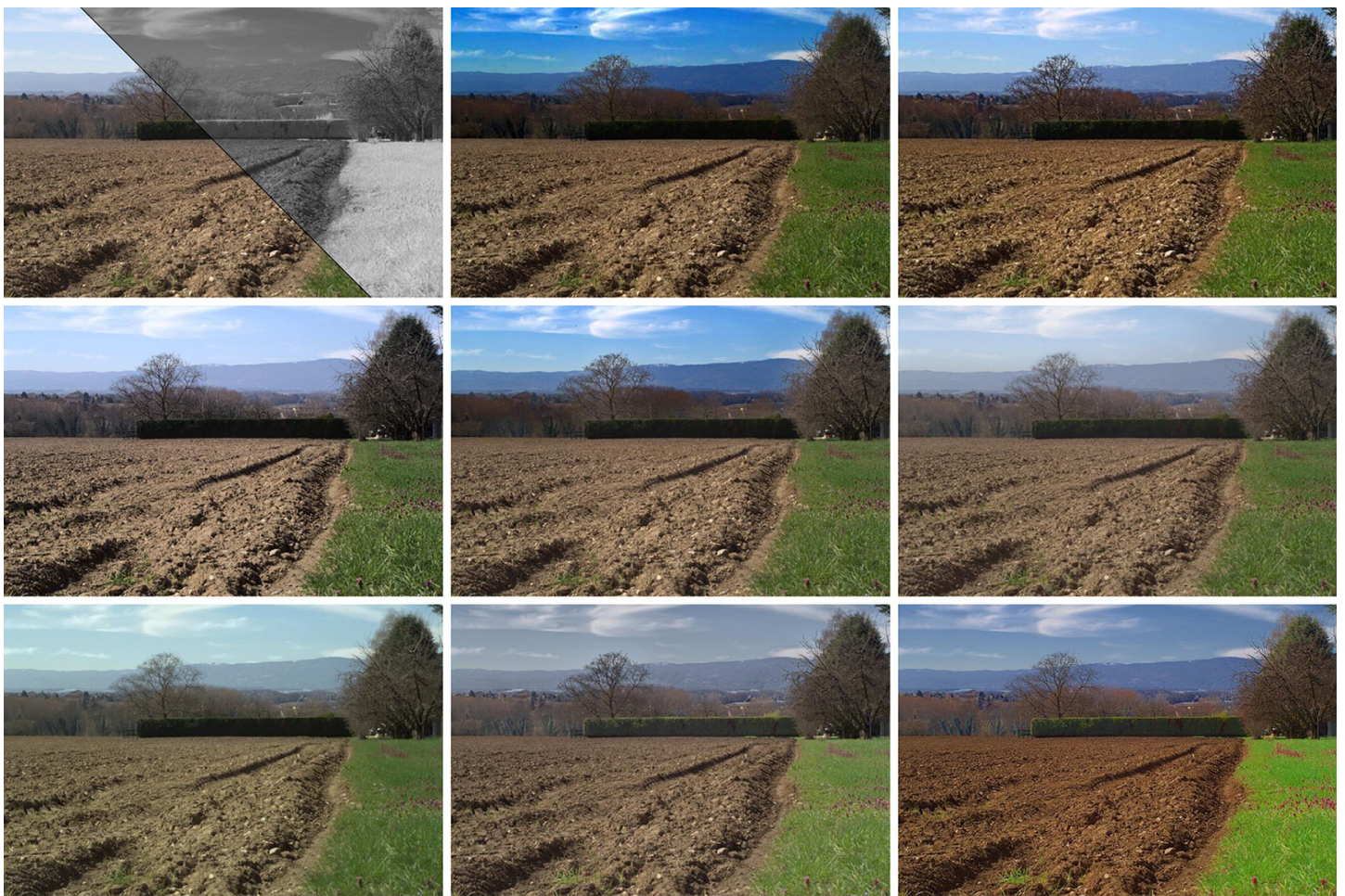

(a)
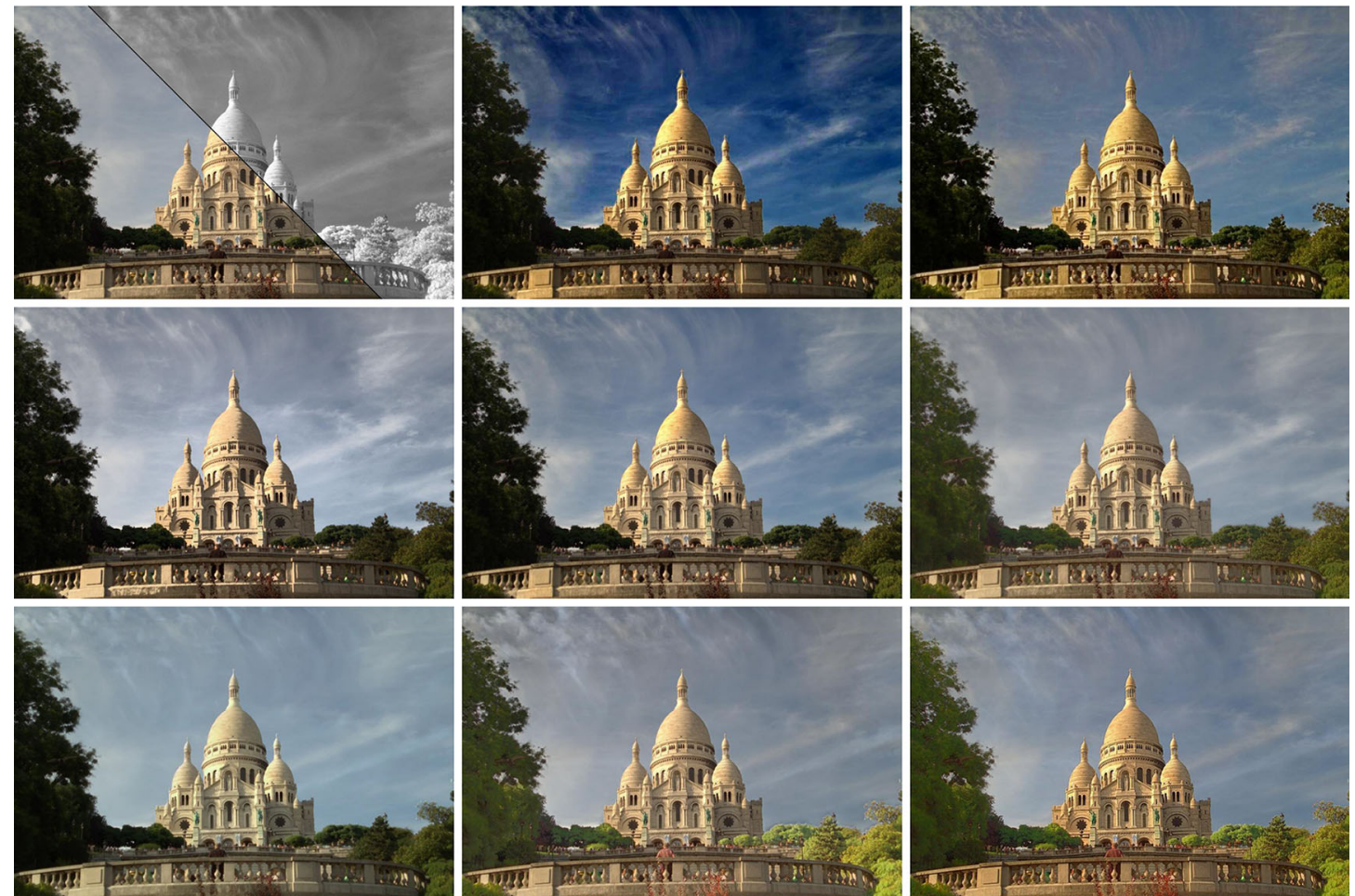

(b)

Figure 6. Demonstration of results for haze-free conditions with sky. For each dataset, 1st row left to right: visible-NIR image pair, results of He et al [7] and results of Tarel and Hautière [9]; 2nd row left to right: results of Ancuti and Ancuti [23], results of Zhu et al [21] and results of Zhang et al [12]; 3rd row left to right: results of Schaul et al [16], our result without CSC and our results with CSC. (Images courtesy: EPFL database [4].) (a) Dataset 5, (b) Dataset 6. 

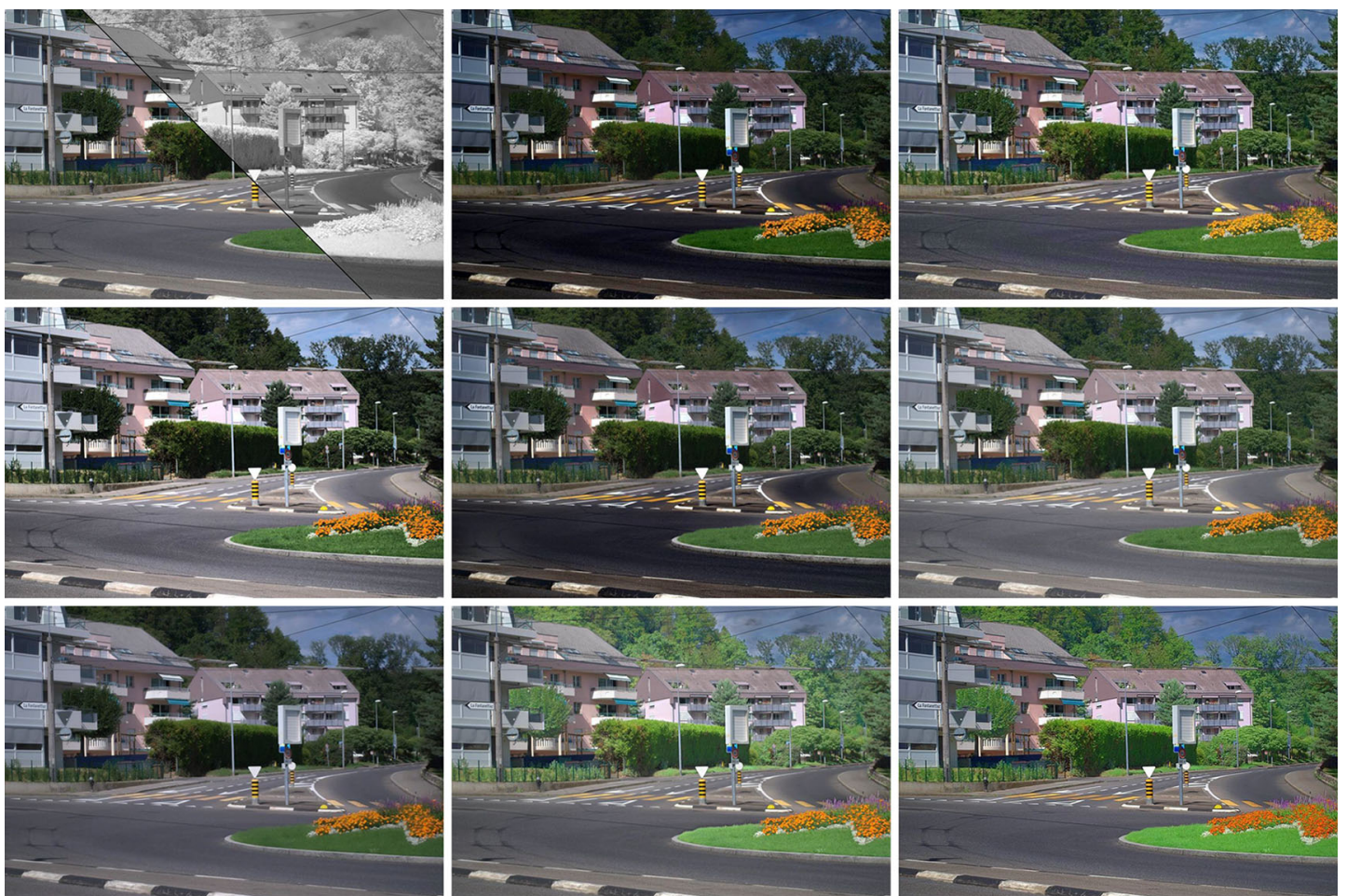

(a)
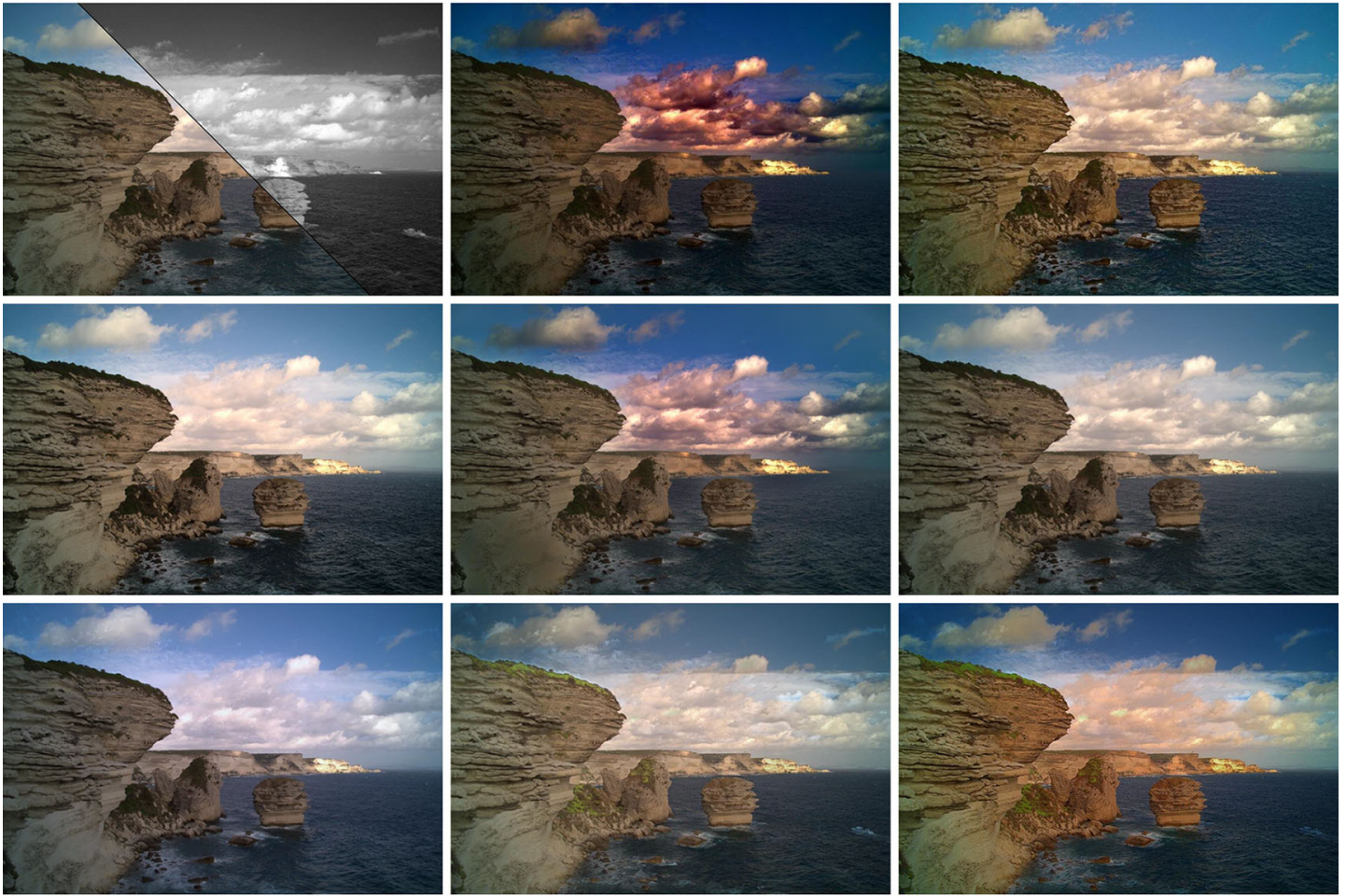

(b)

Figure 7. Demonstration of results for haze-free conditions with sky. For each dataset, 1st row left to right: visible-NIR image pair, results of $\mathrm{He}$ et al [7] and results of Tarel and Hautière [9]; 2nd row left to right: results of Ancuti and Ancuti [23] and results of Zhu et al [21] and results of Zhang et al [12]; 3rd row left to right: results of Schaul et al [16], our result without CSC and our results with CSC. (Images courtesy: EPFL database EPFL [4].) (a) Dataset 7, (b) Dataset 8. 

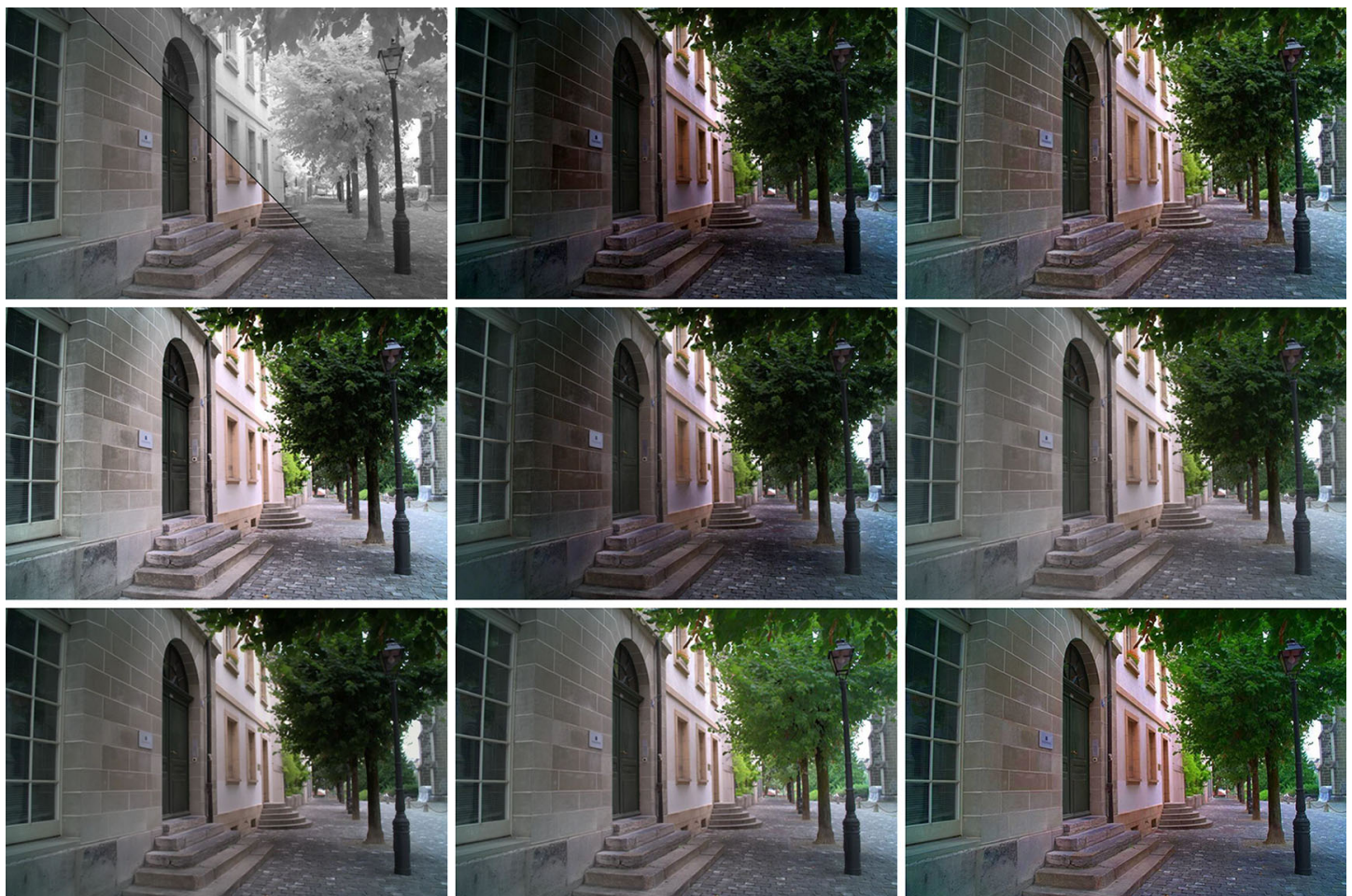

(a)
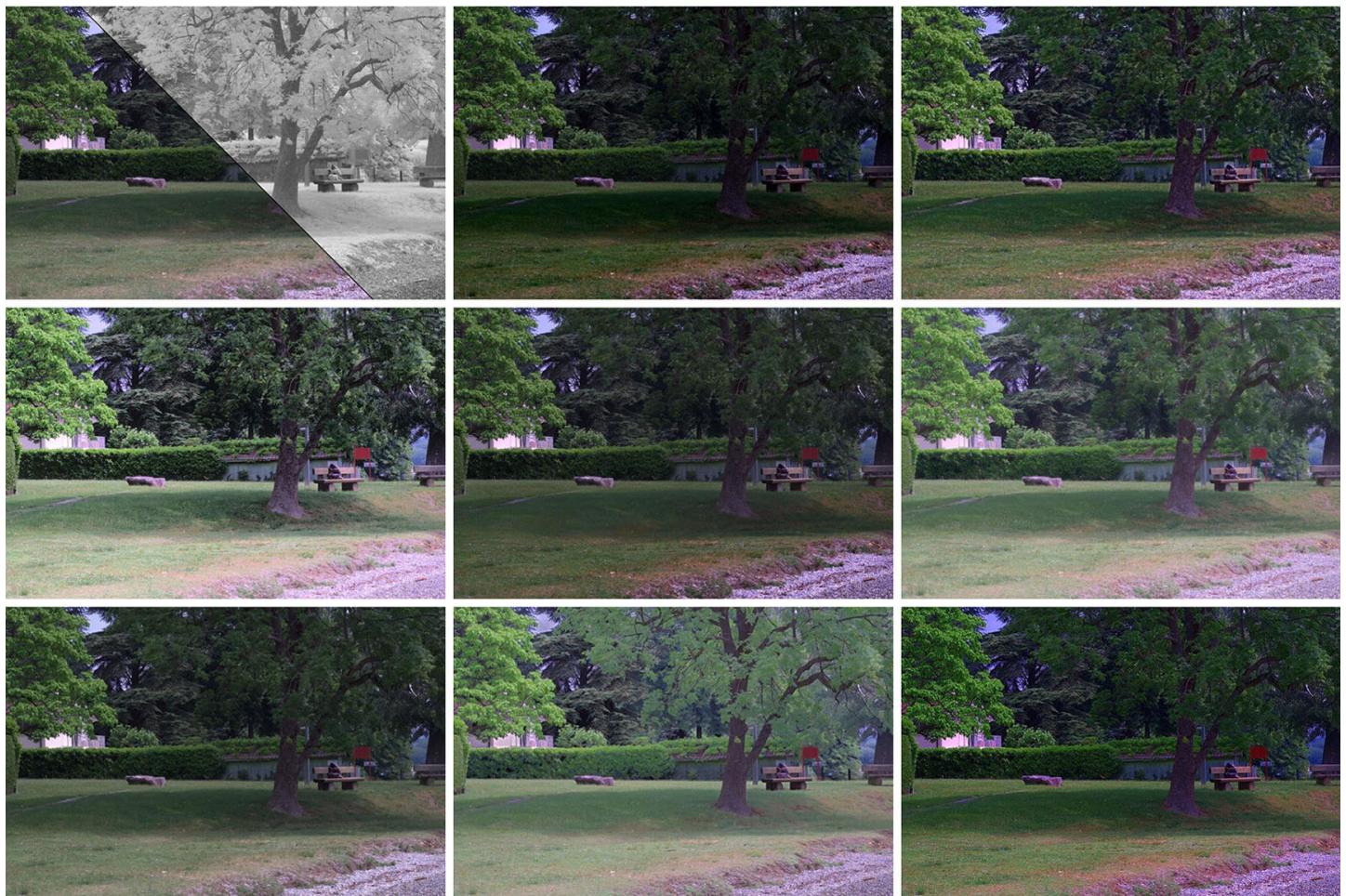

(b)

Figure 8. Demonstration of results for haze-free conditions without sky. For each dataset, 1st row left to right: visible-NIR image pair, results of He et al [7] and results of Tarel and Hautière [9]; 2nd row left to right: results of Ancuti and Ancuti [23], results of Zhu et al [21] and results of Zhang et al [12]; 3rd row left to right: results of Schaul et al [16], our result without CSC and our results with CSC. (Images courtesy: EPFL database [4].) (a) Dataset 9, (b) Dataset 10. 

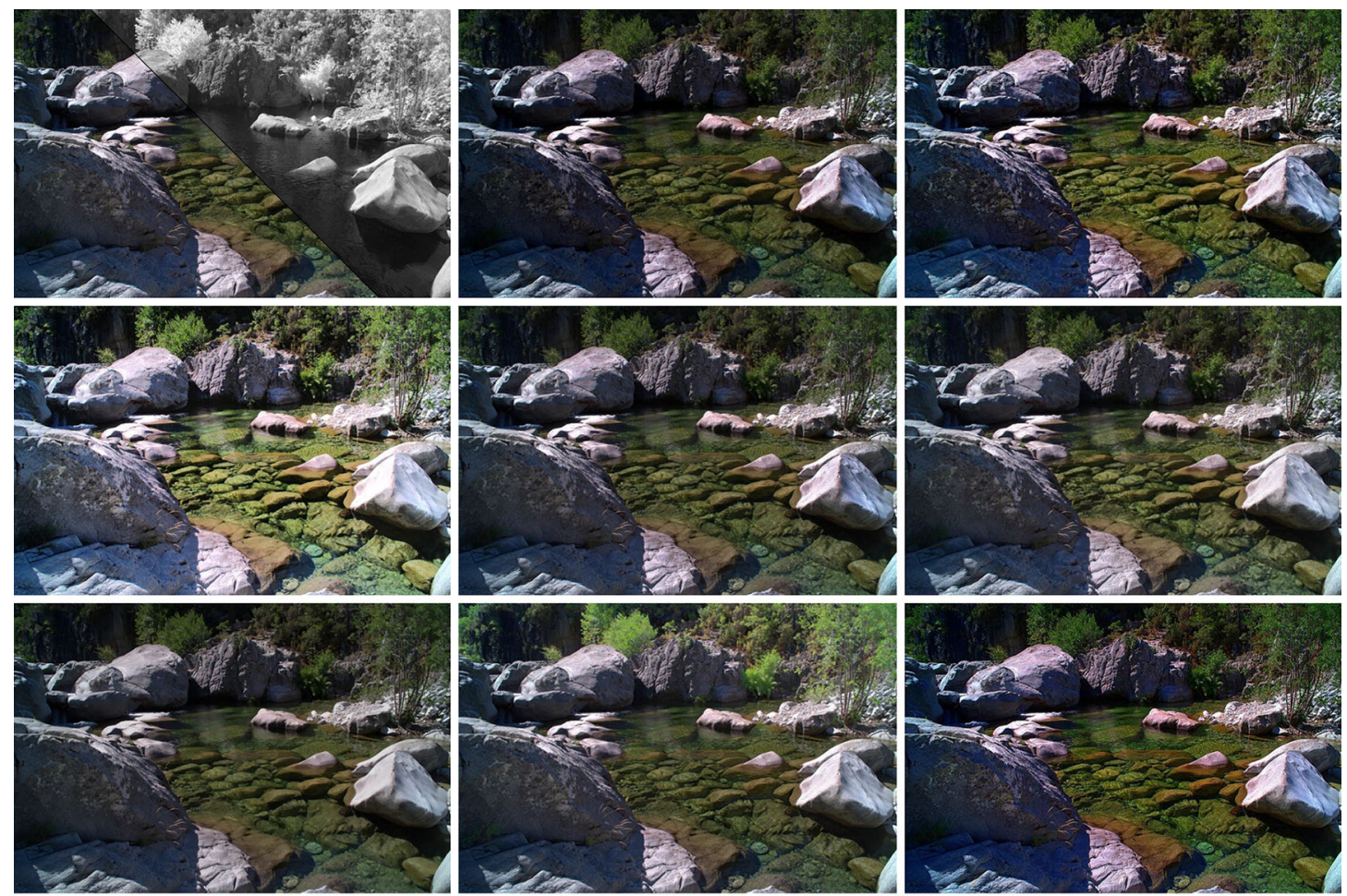

(a)
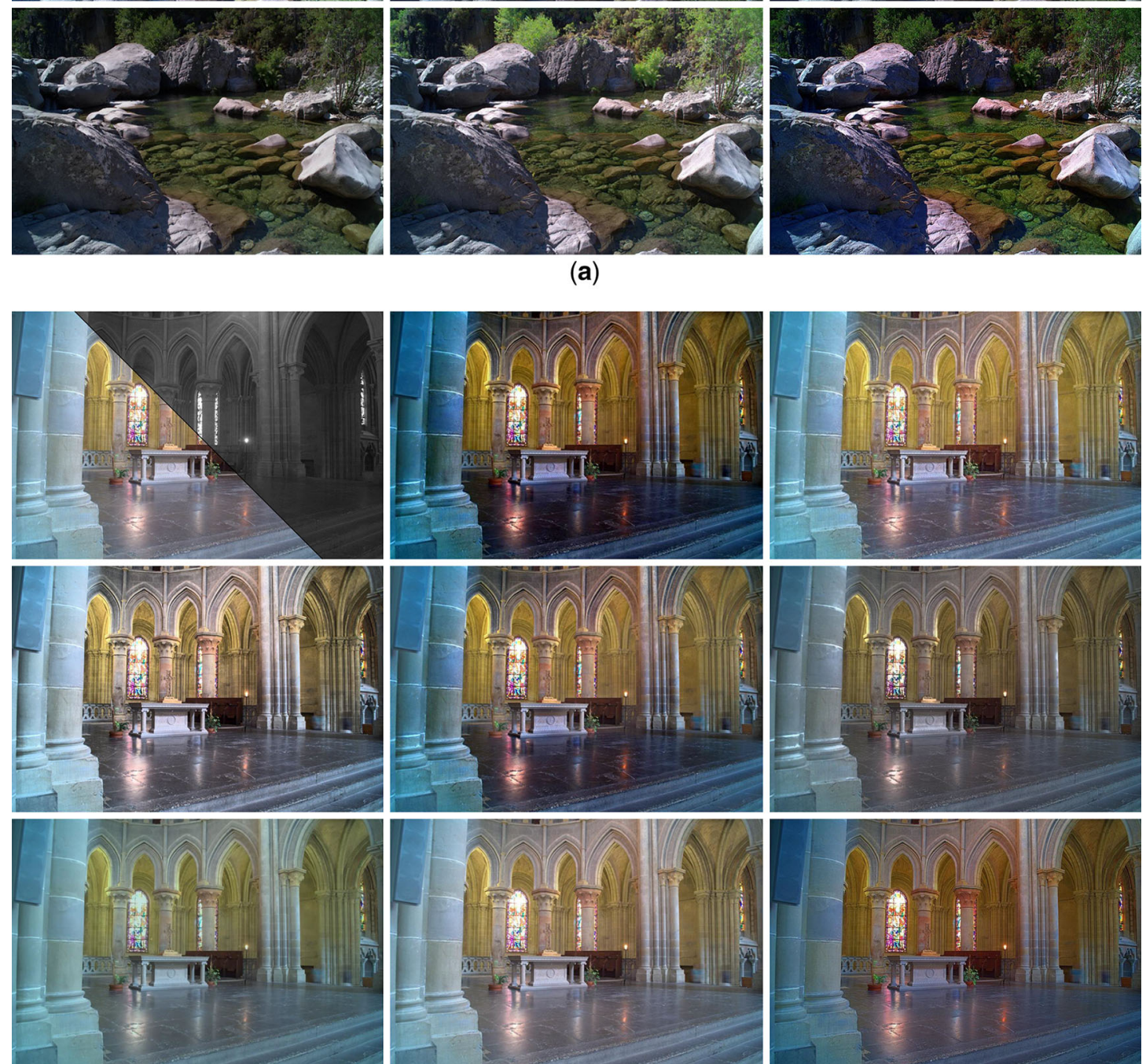

(b)

Figure 9. Demonstration of results for presence water body and indoor image. For each dataset, 1st row left to right: visible-NIR image pair, results of He et al [7], results of Tarel and Hautière [9]; 2nd row left to right: results of Ancuti and Ancuti [23], results of Zhu et al [21], results of Zhang et al [12]; 3rd row left to right: results of Schaul et al [16], our result without CSC and our results with CSC. (Images courtesy: EPFL database [4].) (a) Dataset 11, (b) Dataset 12. 
Table 1. Average execution time for different algorithms.

\begin{tabular}{lc}
\hline Algorithm & Average execution time (s) \\
\hline He et al [7] & 870.3138 \\
Tarel and Hautière [9] & 6.5883 \\
Ancuti and Ancuti [23] & 4.1832 \\
Zhu et al [21] & 25.1972 \\
Zhang et al [12] & 9.5045 \\
Schaul et al [16] & 162.4391 \\
Our algorithm without CSC & 3.1906 \\
Our algorithm with CSC & 3.7667 \\
\hline
\end{tabular}

When compared with these algorithms, the visibility is considerably improved in our results, both with and without CSC. The results of the proposed algorithm contain maximum details and texture in the hazy conditions compared with all the other results. Figure 10 shows cropped portions of the results of different algorithms for distant hazy objects for datasets 1 and 4. One can clearly see that our results, both with and without CSC, exhibit maximum visibility for the hazy regions. This improved visibility is due to the nature of the weight maps generated by our algorithm. Figure 11 shows the corresponding weight maps for datasets 1 and 4 . Here, one can clearly observe that, for visible images, our algorithm gives very less weight for the hazeaffected areas and at the same time gives significant weight for the areas in foreground, which are not affected by haze. For NIR images, a significant weight is given to the hazy areas, which are captured better in NIR spectrum because of less scattering. This ensures that the maximum details are transferred from NIR image to the fused image. The final output images are vivid in colour and sharpness as a result of post-processing procedure, which enhances the perceptual quality of the final results. The performance is equally good for all the categories of the images, making it suitable for both indoor and outdoor applications. Furthermore, in our results, no halo artifacts are observed. The code and full-resolution results can be viewed at https:// drive.google.com/file/d/0B-hGkOHjv3gzVnU5Slg2YWZR WVE/view? usp=sharing. All the results are with the same
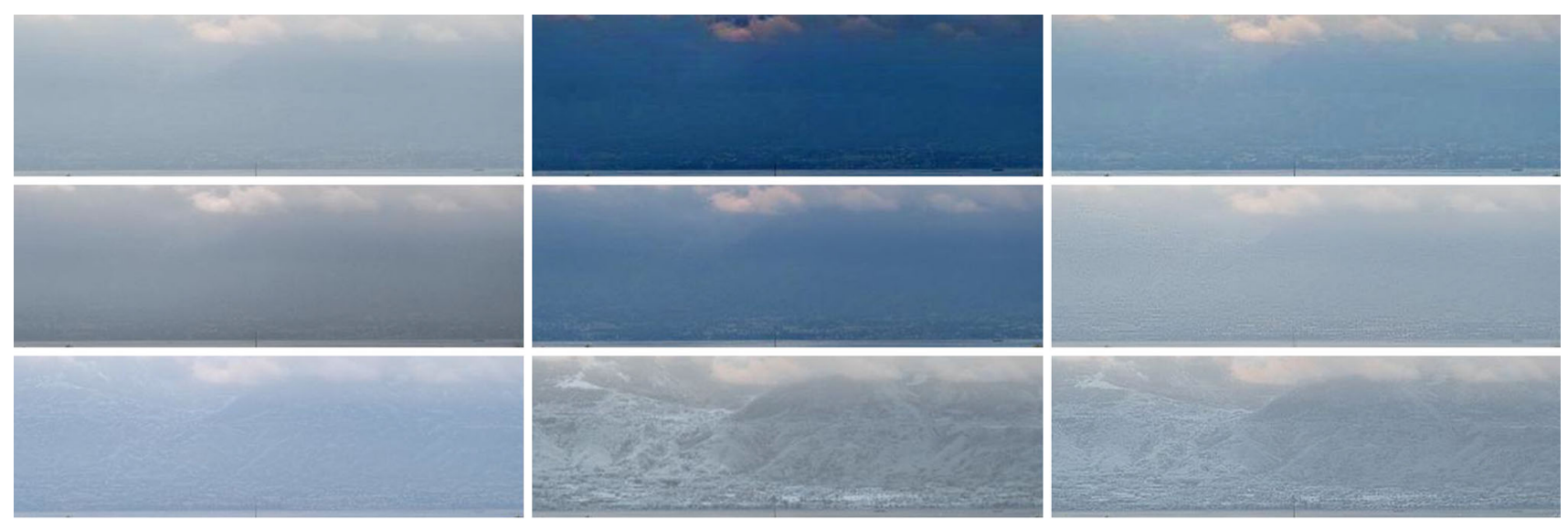

(a)
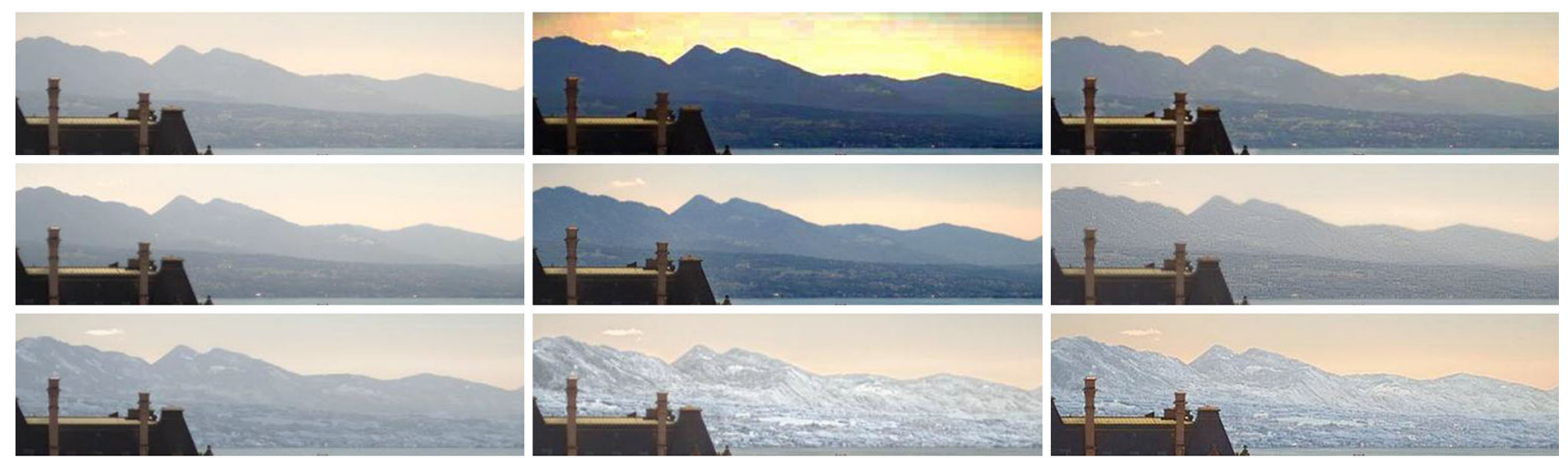

(b)

Figure 10. Cropped portions of the results for distant hazy objects. For each dataset, 1st row left to right: visible image, results of He et al [7] and results of Tarel and Hautière [9]; 2nd row left to right: results of Ancuti and Ancuti [23], results of Zhu et al [21] and results of Zhang et al [12]; 3rd row left to right: results of Schaul et al [16], our result without CSC and our results with CSC. (Images courtesy: EPFL database [4].) (a) Dataset 1, (b) Dataset 4. 

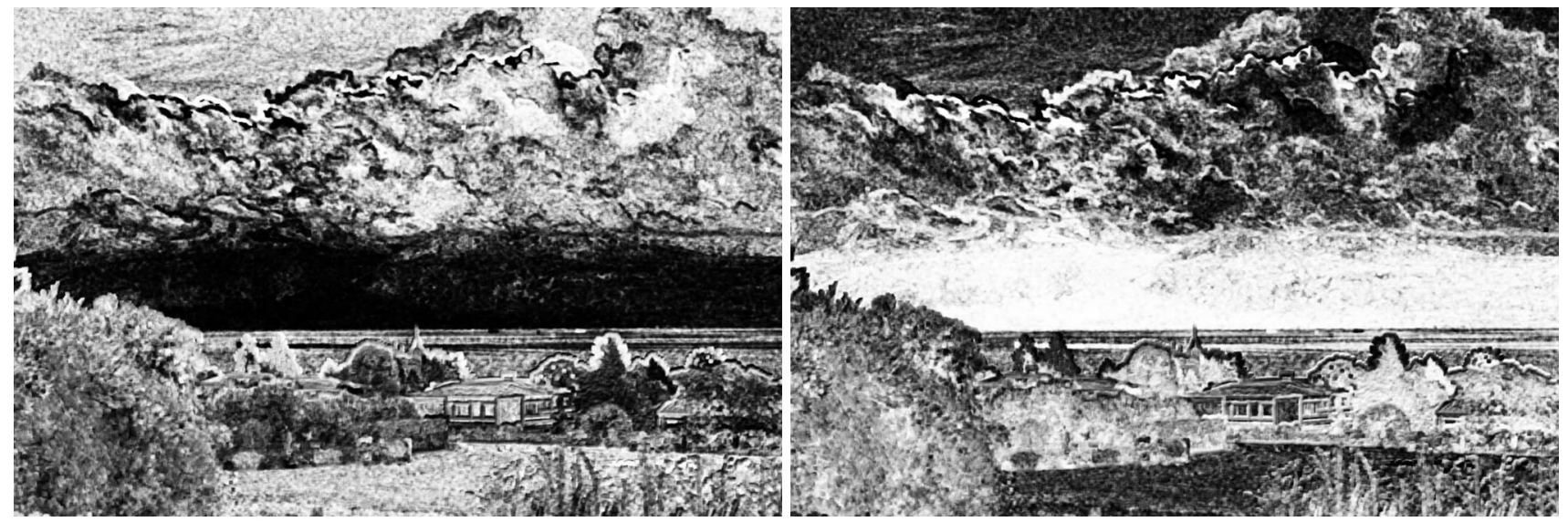

(a)
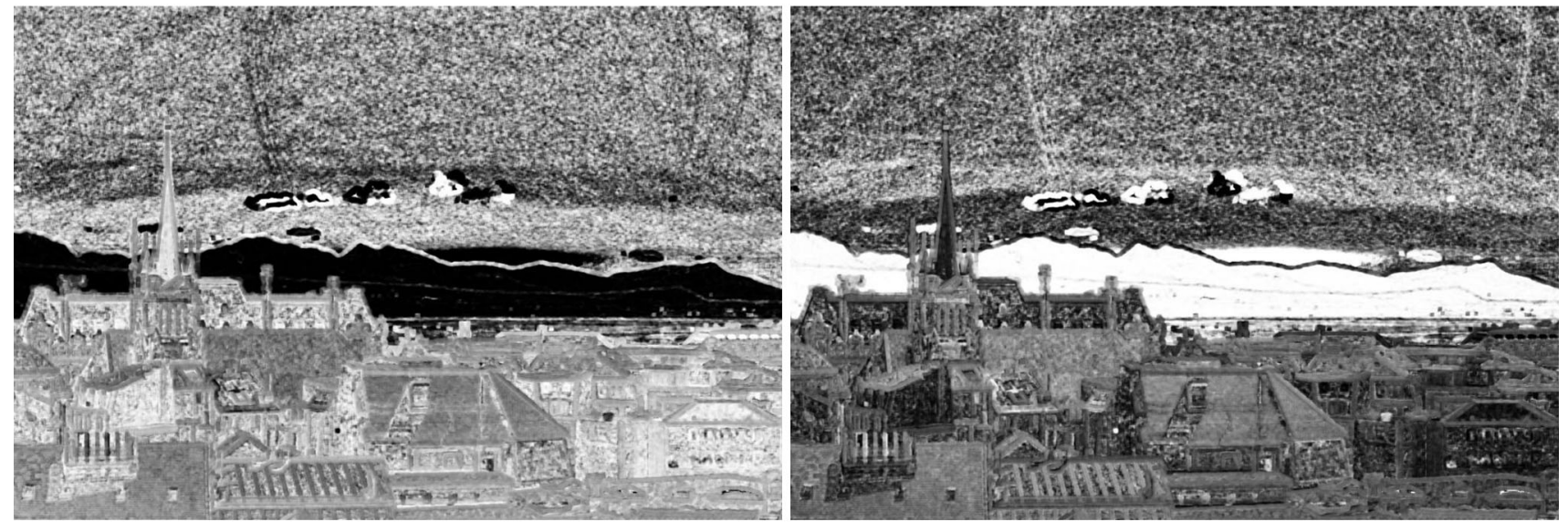

(b)

Figure 11. Final weight maps. For each dataset, left to right: weight map for visible image and weight map for NIR image. (Images courtesy: EPFL database [4].) (a) Dataset 1, (b) Dataset 4.

fixed parameters as defined in each section and do not require modification to obtain good appearance as in the case with the other algorithms.

\section{Quantitative performance}

To validate the visual observations, we perform a quantitative analysis of these results with a variety of quantitative performance indicators. Since, no ground truth is available, no reference image quality indicators are used for quantitative analysis.

The first indicator is the average local entropy. Local entropy of each colour channel is calculated using Eq. (3), and is then averaged to quantify the information content of the image. The results for this assessment are given in table 2. The second indicator is the quality assessment model proposed by Wang et al [27]. Their model calculates the quality of the image by calculating horizontal and vertical features. The results for this assessment are given in table 3. The third indicator is Anisotropic Quality
Index (AQI) proposed by Gabarda and Cristóbal [28]. Their method is based on measuring the variance of the pixel-wise directional entropy of the image upon a set of predefined directions. The results for this assessment are given in table 4 . The fourth indicator proposed by Xue et al [29] is based on quality-aware clustering (QAC). They used the QAC method to learn a set of quality-aware centroids and use them as the codebook to infer the quality of an image patch so that the quality of the whole image can be determined. The results for this assessment are given in table 5 .

The entries in tables 2,4 and 5 indicate that our algorithm without CSC performs moderately compared with other algorithms. This is mainly because of the fact that these quality indicators depends on entropy/variance in a certain way. The multi-resolution fusion employed by us has inherent smoothing effect, which results in reduction of entropy/variance of the fused image. The sharpening process introduced overcomes this drawback, improving the output quality significantly. The results with CSC score are maximum in most of the datasets for these indicators. The 


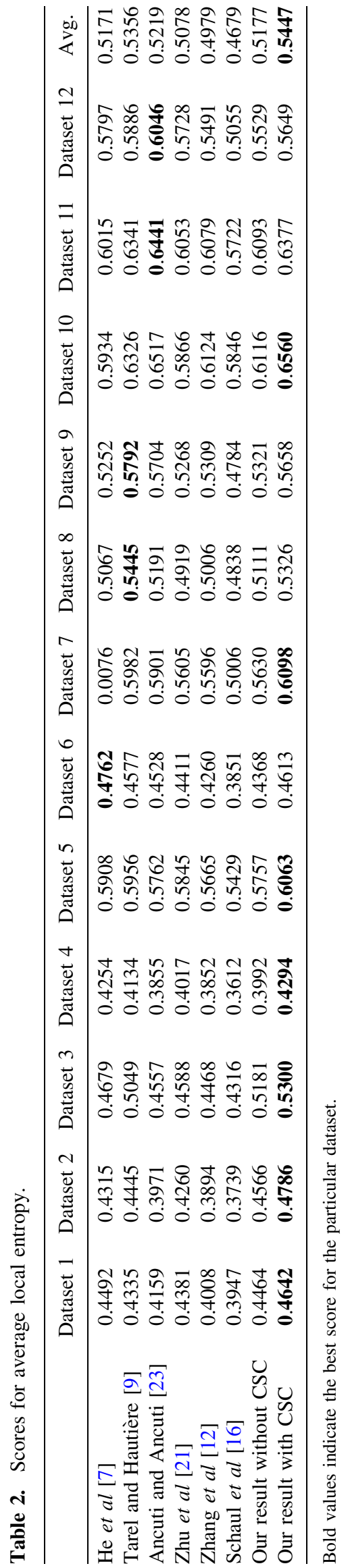

average score of our algorithm with CSC is the best in all these three indicators.

The scores of quality assessment model proposed by Wang et al [27] in table 3 indicate that our algorithm without CSC has either superior or at par performance for all the datasets compared with other outputs. The average score is also substantially better than that from other methods. This is mainly due to the fact that this indicator gives the quality score based on the perceptual quality, which is very much similar to human visual system. After CSC, the performance scores of our algorithm are further improved, outperforming the other algorithms with a substantial margin.

We have treated the dehazing problem as an image fusion of visible and NIR images. Hence, we use two indicators that quantify the quality of the fusion based on the input images that are used for fusion. Accordingly, the fifth indicator used is for amount of edge information proposed by Xydeas and Petrovic [30]. It calculates the amount of edge information transferred from input images to the final fused output. The results for this assessment are given in table 6 . The last indicator proposed by Han et al [31] is based on visual information fidelity (VIFF). The results for this assessment are given in table 7. Last two indicators cannot be used for the results of He et al [7], Tarel and Hautière [9], Ancuti and Ancuti [23] and Zhu et al [21] since their methods are based on single-image dehazing.

In table 6, our algorithm without CSC scores the best almost in all the datasets, indicating that it has maximum amount of edges and in turn details transferred from input images to the output. After CSC, these edges might get modified, and hence, there is a slight decrease in the scores, which are still better than those from the other algorithms. Also, for the VIFF indicator in table 7, our algorithm without CSC has the best scores in almost all the datasets. These scores are improved for the results with CSC.

The scores in tables 2, 3, 4, 5, 6 and 7 clearly indicate that the proposed method performs superior in most of the cases, yielding the highest scores as an individual dataset. When averaged, our results either with or without CSC obtain the highest average score for all the six indicators. This validates the subjective analysis indicating superior performance of our results.

\section{Conclusion}

We have proposed a visible and NIR image fusion algorithm for improved scene visibility. The proposed algorithm does not depend on the haze imaging model. The algorithm is driven by weight maps, calculated on the basis of local entropy, local contrast and visibility. A LaplacianGaussian pyramid based multi-resolution fusion mechanism is used for seamless fusion. A post-processing 

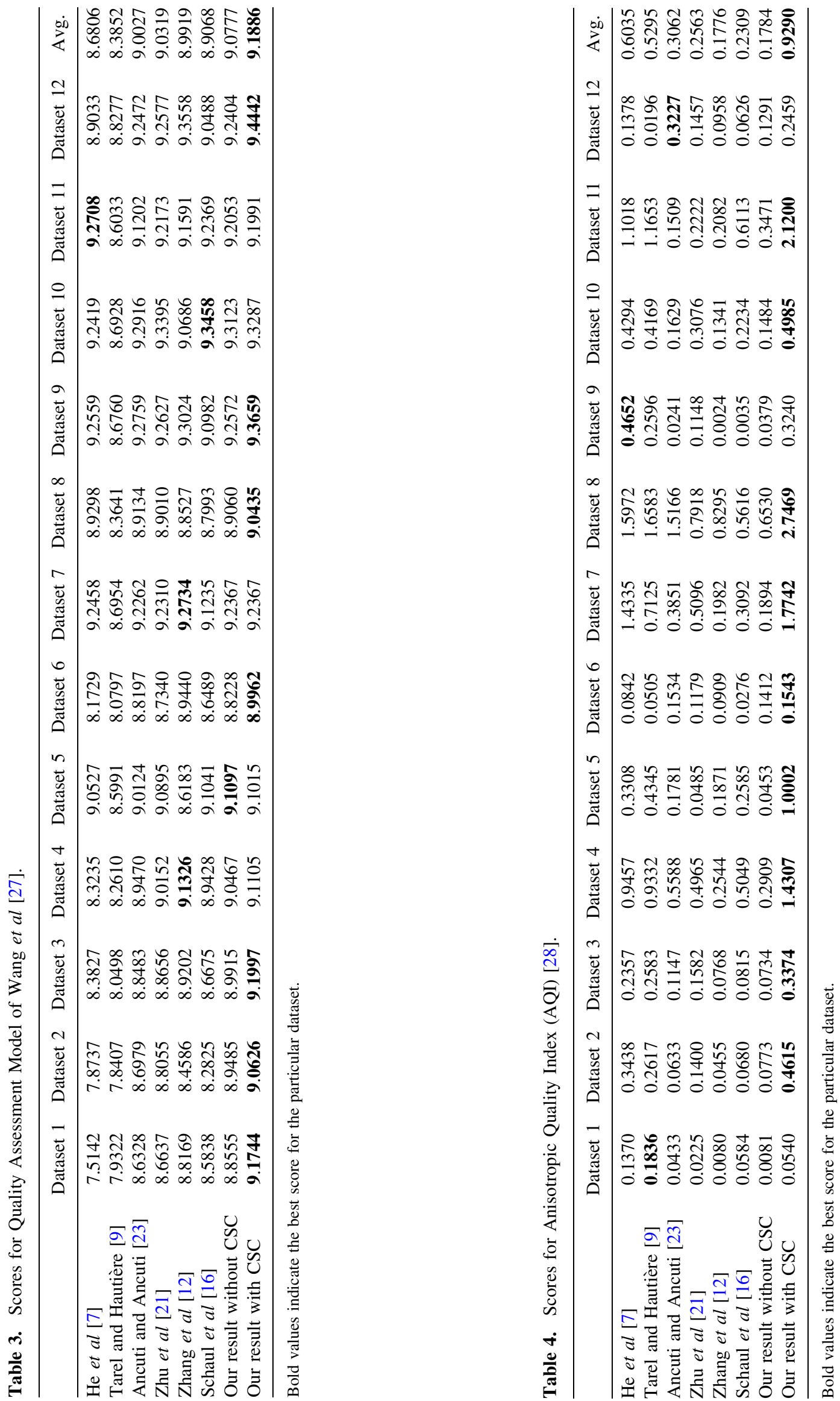

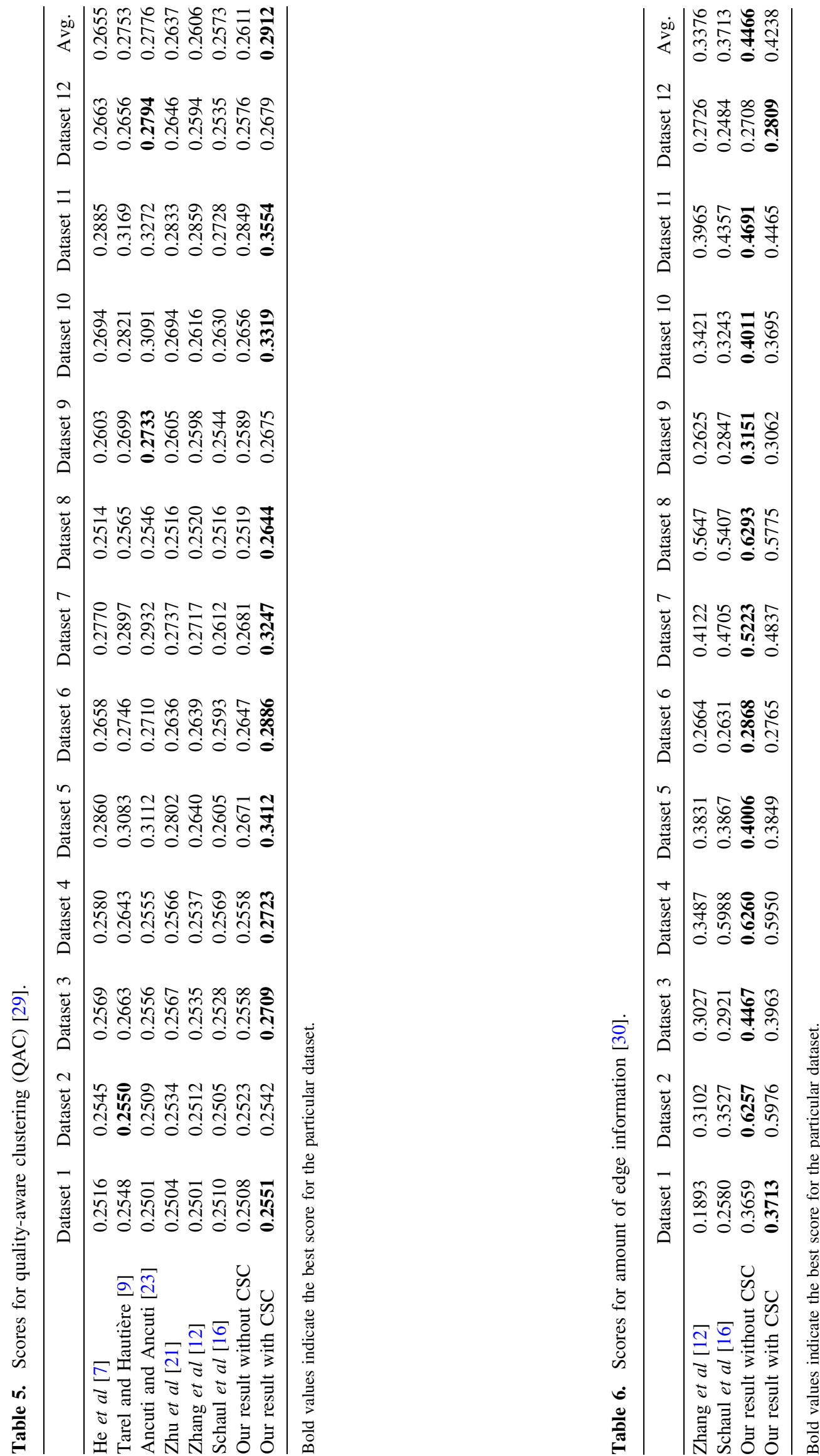
mechanism is added to overcome the shortcomings of the fusion mechanism. The results of our algorithm exhibit excellent details, contrast and colour perception with less computational time. The experimental results clearly indicate that our algorithm performs better than single as well as multi-image dehazing algorithms, in subjective as well as quantitative analysis. The proposed algorithm is fully automatic and does not require any human intervention. Also, our algorithm is not restricted only for haze removal, and can be applied to any images, indoor/outdoor and/or hazy/haze-free situations for improving the scene visibility.

\section{References}

[1] Nayar S K and Narasimhan S G 1999 Vision in bad weather. In: Proceedings of the 7th IEEE International Conference on Computer Vision, 1999, vol. 2, pp. 820-827, doi:10.1109/ ICCV.1999.790306, http://ieeexplore.ieee.org/stamp/stamp. jsp?tp=\&arnumber $=790306$

[2] Schechner Y Y, Narasimhan S G and Nayar S K 2001 Instant dehazing of images using polarization. In: Proceedings of the IEEE Computer Society Conference on Computer Vision and Pattern Recognition, CVPR 2001, vol. 1, pp. I-325-I-332, doi:10.1109/CVPR.2001.990493, http://ieeexplore.ieee.org/ stamp/stamp.jsp?tp=\&arnumber $=990493$

[3] Shwartz S, Namer E and Schechner Y Y 2006 Blind haze separation. In: Proceedings of the IEEE Computer Society Conference on Computer Vision and Pattern Recognition, CVPR 2006, vol. 2, pp. 1984-1991, doi:10.1109/CVPR. 2006.71, http://ieeexplore.ieee.org/stamp/stamp.jsp?tp= \&arnumber $=1640996$

[4] EPFL 2015 EPFL database. Available at: http://ivrg.epfl.ch/ supplementary_material/cvpr11/index.html Last accessed in November 2015

[5] Fattal R 2008 Single image dehazing. ACM Trans. Graph. 27(3): 988-992, doi:10.1145/1360612.1360671, http://doi. acm.org/10.1145/1360612.1360671

[6] Guo F, Tang J and Cai Z X 2014 Image dehazing based on haziness analysis. Int. J. Autom. Comput. 11(1): 78-86, doi:10.1007/s11633-014-0768-7, http://www.ijac.net/EN/ abstract/article_1398.shtml

[7] He K M, Sun J and Tang X O 2009 Single image haze removal using dark channel prior. In: Proceedings of the IEEE conference on Computer Vision and Pattern Recognition, CVPR 2009, pp. 1956-1963, doi:10.1109/CVPR.2009. 5206515, http://ieeexplore.ieee.org/stamp/stamp.jsp?tp= \&arnumber $=5206515$ \&isnumber $=5206488$

[8] Tan R T 2008 Visibility in bad weather from a single image. In: Proceedings of the IEEE Conference on Computer Vision and Pattern Recognition, CVPR 2008, pp. 1-8, doi:10.1109/ CVPR.2008.4587643, http://ieeexplore.ieee.org/stamp/ stamp.jsp?tp $=\&$ arnumber $=4587643 \&$ isnumber $=4587335$

[9] Tarel J P and Hautière N 2009 Fast visibility restoration from a single color or gray level image. In: Proceedings of the IEEE International Conference on Computer Vision, ICCV'09, pp. 2201-2208, doi:10.1109/ICCV.2009.5459251, $\mathrm{http} / / /$ ieeexplore.ieee.org/stamp/stamp.jsp?tp=\&arnumber= $5459251 \&$ isnumber $=5459144$ 
[10] Zhang Y Q, Ding Y, Xiao J S, Liu J and Guo Z 2012 Visibility enhancement using an image filtering approach. EURASIP J. Adv. Signal Process. 2012(1): 220, doi:10.1186/ 1687-6180-2012-220, http://asp.eurasipjournals.com/content/ 2012/1/220

[11] Fredembach C and Süsstrunk S 2008 Colouring the near infrared. In: Proceedings of the IS\&T /SID 16th Color Imaging Conference, Portland, USA, pp. 176-182, http:// infoscience.epfl.ch/record/129419/files/IR_colour.pdf

[12] Zhang X, Sim T and Miao X 2008 Enhancing photographs with near infrared images. In: Proceedings of the IEEE Conference on Computer Vision and Pattern Recognition, CVPR 2008, pp. 1-8, doi:10.1109/CVPR.2008.4587825, http://ieeexplore.ieee.org/stamp/stamp.jsp?tp=\&arnumber $=$ 4587825\&isnumber $=4587335$

[13] Zhuo S, Zhang X, Miao X and Sim T 2010 Enhancing low light images using near infrared flash images. In: Proceedings of the 17th IEEE International Conference on Image Processing, ICIP 2010, pp. 2537-2540, doi:10.1109/ICIP. 2010.5652900, http://ieeexplore.ieee.org/stamp/stamp. jsp?tp=\&arnumber $=5652900 \&$ isnumber $=5648792$

[14] Sadeghipoor Z, Lu Y M and Süsstrunk S 2011 Correlationbased joint acquisition and demosaicing of visible and nearinfrared images. In: Proceedings of the 18th IEEE International Conference on Image Processing, ICIP 2011, Brussels, Belgium, pp. 3165-3168, doi:10.1109/ICIP.2011. 6116339, http://dblp.uni-trier.de/db/conf/icip/icip2011. html\#SadeghipoorLS11

[15] Feng C, Zhuo S, Zhang X, Shen L and Süsstrunk S 2013 Near-infrared guided color image dehazing. In: Proceedings of the 20th IEEE International Conference on Image Processing, ICIP 2013, pp. 2363-2367, doi:10.1109/ICIP.2013. 6738487, http://infoscience.epfl.ch/record/188639/files/Ima geDehazing_icip2013.pdf

[16] Schaul L, Fredembach C and Süsstrunk S 2009 Color image dehazing using the near-infrared. In: Proceedings of the 16th IEEE International Conference on Image Processing, ICIP 2009, pp. 1629-1632, doi:10.1109/ICIP.2009.5413700, http://ieeexplore.ieee.org/stamp/stamp.jsp?tp=\&arnumber= 5413700 \&isnumber $=5413332$

[17] Long J, Shi Z, Tang W and Zhang C 2014 Single remote sensing image dehazing. Geosci. Remote Sens. Lett. IEEE 11(1): 59-63, doi:10.1109/LGRS.2013.2245857, http:// ieeexplore.ieee.org/stamp/stamp.jsp?tp=\&arnumber= 6476634

[18] Makarau A, Richter R, Muller R and Reinartz P 2014 Haze detection and removal in remotely sensed multispectral imagery. IEEE Trans. Geosci. Remote Sensing 52(9): 5895-5905, doi:10.1109/TGRS.2013.2293662, http://ieeex plore. ieee.org/stamp/stamp.jsp?tp=\&arnumber $=6697865$

[19] Lan X, Zhang L, Shaen H, Yuan Q and Li H 2013 Single image haze removal considering sensor blur and noise. EURASIP J. Adv. Signal Process. 2013: 86, doi:10.1186/ 1687-6180-2013-86, http://asp.eurasipjournals.com/content/ pdf/1687-6180-2013-86.pdf
[20] Fattal R 2014 Dehazing using color-lines. ACM Trans. Graph. 34(1): 13:1-13:14, doi:10.1145/2651362, http://doi. acm.org/10.1145/2651362

[21] Zhu Q, Mai J and Shao L 2015 A fast single image haze removal algorithm using color attenuation prior. IEEE Trans. Image Process. 24(11): 3522-3533, doi:10.1109/TIP.2015. 2446191, http://ieeexplore.ieee.org/stamp/stamp.jsp?tp= \&arnumber $=7128396$

[22] Sulami M, Geltzer I, Fattal R and Werman M 2014 Automatic recovery of the atmospheric light in hazy images. In: Proceedings of the IEEE International Conference on Computational Photography, ICCP 2014, pp. 1-11, doi:10. 1109/ICCPHOT.2014.6831817, http://dx.doi.org/10.1109/ ICCPHOT.2014.6831817

[23] Ancuti C O and Ancuti C 2013 Single image dehazing by multi-scale fusion. IEEE Trans Image Process. 22(8): 3271-3282, doi:10.1109/TIP.2013.2262284, http://dx.doi. org/10.1109/TIP.2013.2262284

[24] Ancuti C and Ancuti C O 2014 Effective contrast-based dehazing for robust image matching. IEEE Geosci. Remote Sensing Lett. 11(11): 1871-1875, doi:10.1109/LGRS.2014. 2312314, http://dx.doi.org/10.1109/LGRS.2014.2312314

[25] Mertens T, Kautz J and Reeth F V 2007 Exposure fusion. In: Proceedings of the 15th Pacific Conference on Computer Graphics and Applications, $P G$ '07, IEEE Computer Society, Los Alamitos, CA, USA, pp. 382-390, doi:10.1109/PG.2007. 17, http://ieeexplore.ieee.org/stamp/stamp.jsp?tp=\&arnum ber $=4392748$

[26] Bovik A 2009 The essential guide to image processing. Academic Press, Boston, USA (chap 12), pp. 263-291

[27] Wang Z, Sheikh H R and Bovik A C 2002 No-reference perceptual quality assessment of JPEG compressed images. In: Proceedings of the IEEE International Conference on Image Processing, ICIP 2002, vol.1, pp. I-477-I-480, doi:10. 1109/ICIP.2002.1038064, http://ieeexplore.ieee.org/stamp/ stamp.jsp?tp=\&arnumber $=1038064$ \&isnumber $=22252$

[28] Gabarda S and Cristóbal G 2007 Blind image quality assessment through anisotropy. J. Opt. Soc. Am. 24(12): B42-B51, doi:10.1364/JOSAA.24.000B42, http://digital. csic.es/bitstream/10261/61694/1/Gabarda.pdf

[29] Xue W, Zhang L and Mou X 2013 Learning without human scores for blind image quality assessment. In: Proceedings of the IEEE Conference on Computer Vision and Pattern Recognition, CVPR 2013, IEEE Computer Society, Washington, DC, USA, pp. 995-1002, doi:10.1109/CVPR.2013. 133, http://dx.doi.org/10.1109/CVPR.2013.133

[30] Xydeas C S and Petrovic V S 2000 Objective pixel-level image fusion performance measure. Proc. SPIE 4051 (Sensor Fusion: Architectures, Algorithms, and Applications IV): 89-98, doi:10.1117/12.381668, http://dx.doi.org/10.1117/12. 381668

[31] Han Y, Cai Y, Cao Y and Xu X 2013 A new image fusion performance metric based on visual information fidelity. Inf. Fusion 14(2): 127-135, doi:10.1016/j.inffus.2011.08.002, http://dx.doi.org/10.1016/j.inffus.2011.08.002 